\title{
A human neutralizing antibody targets the receptor-binding site of SARS-CoV-2
}

https://doi.org/10.1038/s41586-020-2381-y

Received: 2 April 2020

Accepted: 19 May 2020

Published online: 26 May 2020

Check for updates

\author{
Rui Shi ${ }^{1,2,14}$, Chao Shan ${ }^{3,14}$, Xiaomin Duan ${ }^{1,2,14}$, Zhihai Chen ${ }^{4,14}$, Peipei Liu ${ }^{5,14}$, Jinwen Song ${ }^{6,14}$, \\ Tao Song ${ }^{1,7,8}$, Xiaoshan $\mathrm{Bi}^{1,9}$, Chao Han ${ }^{1,2}$, Lianao $\mathrm{Wu}^{9,10}, \mathrm{Ge} \mathrm{Gao}^{3}$, Xue Hu${ }^{3}$, Yanan Zhang ${ }^{3}$, \\ Zhou Tong ${ }^{1,10}$, Weijin Huang ", William Jun Liu ${ }^{5}$, Guizhen Wu ${ }^{5}$, Bo Zhang ${ }^{3}$, Lan Wang", \\ Jianxun Qi ${ }^{10,12}$, Hui Feng ${ }^{13}$, Fu-Sheng Wang ${ }^{6 凶}$, Qihui Wang $^{1,10,12 \bowtie}$, George Fu Gao $^{10 \bowtie}$, \\ Zhiming Yuan $^{3 凶}$ \& Jinghua Yan ${ }^{1,10,12 \bowtie}$
}

\begin{abstract}
An outbreak of coronavirus disease 2019 (COVID-19) ${ }^{1-3}$, caused by severe acute respiratory syndrome coronavirus 2 (SARS-CoV-2) ${ }^{4}$, has spread globally. Countermeasures are needed to treat and prevent further dissemination of the virus. Here we report the isolation of two specific human monoclonal antibodies (termed CA1 and CB6) from a patient convalescing from COVID-19. CA1 and CB6 demonstrated potent SARS-CoV-2-specific neutralization activity in vitro. In addition, CB6 inhibited infection with SARS-CoV-2 in rhesus monkeys in both prophylactic and treatment settings. We also performed structural studies, which revealed that $\mathrm{CB} 6$ recognizes an epitope that overlaps with angiotensin-converting enzyme 2 (ACE2)-binding sites in the SARS-CoV-2 receptor-binding domain, and thereby interferes with virus-receptor interactions by both steric hindrance and direct competition for interface residues. Our results suggest that $\mathrm{CB} 6$ deserves further study as a candidate for translation to the clinic.
\end{abstract}

Emerging and recurrent pathogens are global challenges for public health ${ }^{5}$. Cases of a typical pneumonia-officially designated as COVID-19 by the World Health Organization-have been reported since the end of $2019^{1-3}$. The underlying pathogen has been confirmed to be a novel coronavirus, which-on the basis of phylogenetic evidence-is closely related to a bat coronavirus ${ }^{6}$. The International Committee on Taxonomy of Viruses has recently named the virus SARS-CoV- $2^{4}$, although some virologists have argued that HCoV-19 would be more appropriate ${ }^{7}$. As of 1 April 2020, there were more than 880,000 confirmed cases of COVID-19 and more than 44,000 COVID-19-related deaths globally (https://covid19.who.int/). No vaccines or drugs have yet been approved for the treatment of COVID-19. However, multiple clinical trials have been launched to evaluate the efficacy and safety of inactivated convalescent plasma in the treatment of COVID-19 (www.ClinicalTrials. gov, identifiers NCT04292340, NCT04327349 and NCT04321421). Preliminary data have suggested a potential benefit from treatment with plasma from convalescent individuals (http://www.nhc.gov. cn/xcs/fkdt/202002/2a1d9d8569c549e7a5d5737751c74480.shtml), which indicates that neutralizing antibodies from convalescent patients could inhibit virus infection and have potential for clinical use. However, the practical limitations of collecting plasma from convalescent individuals, and a lack of suitable risk assessment, make large-scale plasma transfusion programmes a challenge.

SARS-CoV-2 is the third highly pathogenic coronavirus that infects humans to have emerged; it uses the same receptorangiotensin-converting enzyme 2 (ACE2)-for cell entry as severe acute respiratory syndrome coronavirus (SARS-CoV) ${ }^{6,8}$. As with other coronaviruses, the SARS-CoV-2 spike (S) protein can be further separated into S1 and S2 subdomains, with S1 binding to the host receptor and S2 mediating membrane fusion ${ }^{9}$. The receptor-binding domain (RBD) of the SARS-CoV-2S protein at the S1C-terminal domain has recently been mapped, and the crystal structure of the SARS-CoV-2 RBD in complex with human ACE2 has been solved ${ }^{10-13}$. Previous studies have revealed that a large number of antibodies show neutralization activity by targeting the RBD of either SARS-CoV or Middle East respiratory syndrome coronavirus (MERS-CoV), presumably by disrupting the virus-receptor engagement ${ }^{14-16}$. Thus, we attempted to isolate neutralizing monoclonal antibodies (mAbs) against SARS-CoV-2 from patients convalescing from COVID-19, using a similar strategy to that previously reported ${ }^{17,18}$.

We used a recombinant RBD of the SARS-CoV-2S protein as the bait to sort specific memory B cells from peripheral blood mononuclear cells of a patient convalescing from COVID-19. The sequences of the

${ }^{1}$ CAS Key Laboratory of Microbial Physiological and Metabolic Engineering, Institute of Microbiology, Chinese Academy of Sciences, Beijing, China. ${ }^{2}$ University of Chinese Academy of

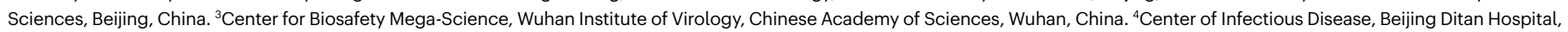
Capital Medical University, Beijing, China. ${ }^{5} \mathrm{NHC}$ Key Laboratory of Biosafety, National Institute for Viral Disease Control and Prevention, Chinese Center for Disease Control and Prevention, Beijing, China. ${ }^{6}$ Treatment and Research Center for Infectious Diseases, The Fifth Medical Center of PLA General Hospital, National Clinical Research Center for Infectious Diseases, Beijing, China. ${ }^{7}$ Shanxi Academy of Advanced Research and Innovation, Taiyuan, China. ${ }^{8}$ College of Animal Science and Technology, Hebei Normal University of Science and Technology, Qinhuangdao, China. ${ }^{9}$ Institute of Physical Science and Information, Anhui University, Hefei, China. ${ }^{10} \mathrm{CAS}$ Key Laboratory of Pathogenic Microbiology and Immunology, Institute of

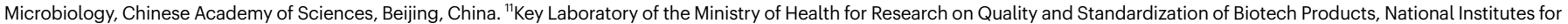
Food and Drug Control, Beijing, China. ${ }^{12}$ Savaid Medical School, University of Chinese Academy of Sciences, Beijing, China. ${ }^{13}$ Shanghai Junshi Biosciences Co. Ltd, Shanghai, China. ${ }^{14}$ These authors contributed equally: Rui Shi, Chao Shan, Xiaomin Duan, Zhihai Chen, Peipei Liu, Jinwen Song. ${ }^{凶}$ e-mail: fswang302@163.com; wangqihui@im.ac.cn; gaof@im.ac.cn; yzm@wh.iov.cn; yanjh@im.ac.cn 


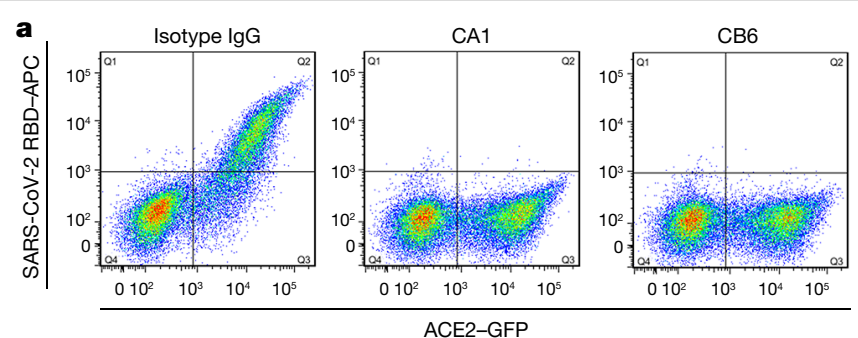

b

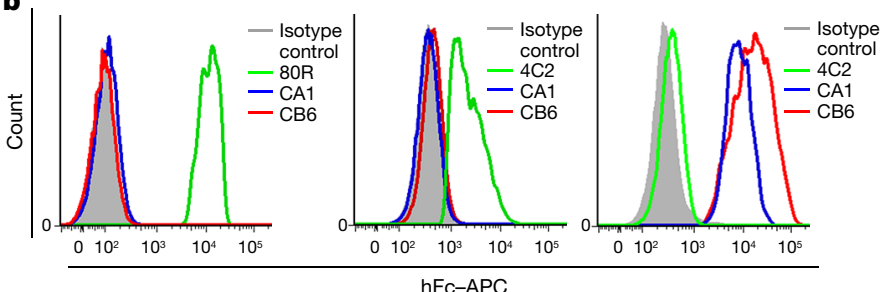

c $\quad K_{\mathrm{D}}=4.68 \pm 1.64 \mathrm{nM}$

$k_{\mathrm{a}}=1.77 \pm 1.57 \times 10^{6}$ per ms
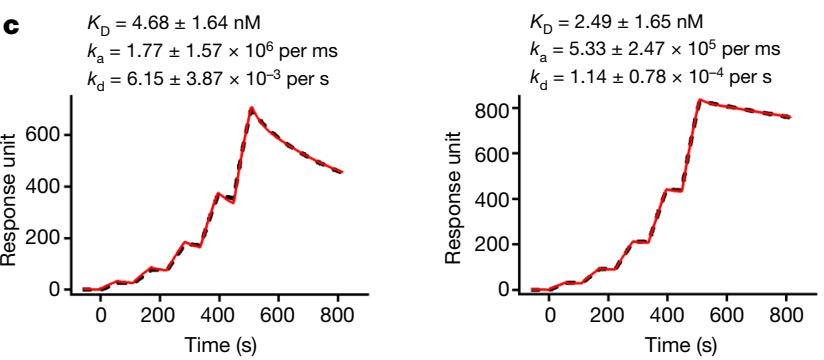

Fig. 1 CA1 and CB6 specifically recognize RBD and block the binding of the SARS-CoV-2 RBD to ACE2. a, CA1 and CB6 can block SARS-CoV-2 RBD binding to ACE2, as shown in a FACS-based assay. ACE2-GFP fusion protein was transiently expressed on the surface of HEK293T cells, and stained with SARS-CoV-2 RBD protein pre-incubated with isotype-control IgG, CA1 or CB6. Experiments were performed twice, and one set of representative data is displayed.b, Left, HEK293T cells that express SARS-CoV S protein were permeabilized, and stained with isotype-control IgG, CA1, CB6 or the positive-control mAb $80 \mathrm{R}^{26}$. Cells expressing MERS-CoVS (middle) or SARS-CoV-2S (right) protein were stained with isotype-control IgG, CA1, CB6 or the control mAb $4 \mathrm{C}^{14}$. Experiments were performed twice, and one set of representative data is displayed. $\mathrm{hFc}$, human $\mathrm{Fc}$. c, The binding kinetics of CA1 (left) and CB6 (right) to recombinant SARS-CoV-2 RBD were obtained using the BIAcore $8 \mathrm{~K}$ system in single-cycle mode. $\mathrm{mAbs}$ were captured on the chip, and serial dilutions of RBD were then injected over the chip surface. The $K_{\mathrm{D}}$ is labelled accordingly. Values are mean \pm s.d. of three independent results.

variable regions of IgG antibodies in the sorted cells were obtained through $5^{\prime}$ rapid amplification of cDNA ends (RACE) from individual B cells. We identified two mAbs - named CA1 and CB6 (Extended Data Table 1) - as being able to block the binding of soluble SARS-CoV-2 RBD to the ACE2 receptor transiently expressed on HEK293T cells (Fig. 1a). Binding analysis of $\mathrm{CA} 1$ and $\mathrm{CB} 6$ by fluorescence-activated cell sorting (FACS) revealed that both $m$ Abs can specifically bind to HEK293T cells transfected with SARS-CoV-2S protein, but not to cells transfected with SARS-CoV S or MERS-CoV S protein (Fig. 1b). We assessed the binding kinetics of CA1 and CB6 using a surface plasmon resonance (SPR) assay. The measured equilibrium constants $\left(K_{\mathrm{D}}\right)$ of CA1 and CB6 with the SARS-CoV-2 RBD were $4.68 \pm 1.64 \mathrm{nM}$ and $2.49 \pm 1.65 \mathrm{nM}$, respectively (Fig. 1c, Extended Data Table 2). Because CA1 and CB6 have distinctive complementarity-determining-region (CDR) sequences (Extended Data Table 1), we examined the potential competitive binding of CA1 and CB6 to the SARS-CoV-2 RBD in an Octet-based binding assay. Recombinant SARS-CoV-2 RBD was first biotinylated and immobilized on a streptavidin biosensor, and then saturated with $\mathrm{CB} 6$ or CA1. The addition of CA1 to CB6-saturated probe or CB6 to CA1-saturated probe showed no complementary binding (Extended Data Fig. 1), which indicates that CA1 and CB6 bind to overlapping epitopes on the SARS-CoV-2 RBD.
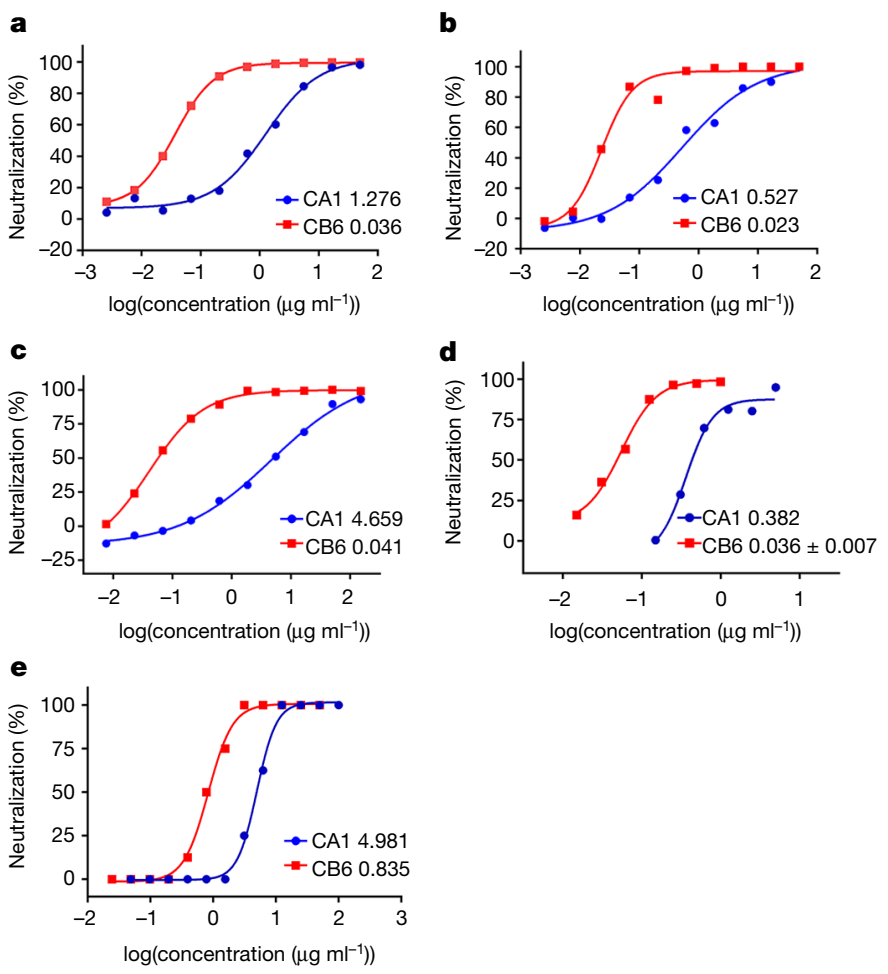

Fig. 2 | CB6 and CA1 can effectively neutralize SARS-CoV-2 pseudovirus or live SARS-CoV-2 virus in vitro. a-c, SARS-CoV-2 pseudovirus was incubated with threefold serially diluted CA1 or CB6. The mixtures were then added to Huh7 (a), Calu-3 (b) or HEK293T (c) cells. After a 24-h incubation, neutralization potencies of the mAbs were evaluated in a luciferase assay system. $\mathrm{ND}_{50}$ values were calculated by fitting the luciferase activity from serially diluted antibody to a sigmoidal dose-response curve. The experiments were performed twice and similar results were obtained. One set of representative data from one experiment is shown; data are average values of two replicates. d, Mixtures of live SARS-CoV-2 virus and serially diluted CA1 or CB6 were added to Vero E6 cells. After a 30-h incubation, $\mathrm{ND}_{50}$ was calculated by fitting the viral RNA copies (determined by quantitative PCR with reverse transcription (RT-qPCR)) from serially diluted antibody to a sigmoidal dose-response curve. The experiments for CA1 were performed twice, and similar results were obtained. For CB6, the values shown are the mean \pm s.d. of three independent experiments. For both CA1 and CB6, one set of representative data from one experiment is shown; data are average values of two replicates. e, Mixtures of SARS-CoV-2 and serially diluted CA1 or CB6 were added to Vero $\mathrm{E} 6$ cells. After a 72 -h incubation, $\mathrm{ND}_{50}$ values were calculated by fitting the proportion of cytopathic effect with serially diluted antibody to a sigmoidal dose-response curve. The experiments were independently performed twice and similar results were obtained. One set of representative data is shown.

Next, we investigated the neutralization abilities of CA1 and CB6 against SARS-CoV-2 infection using pseudoviruses that express $\mathrm{S}$ antigen of SARS-CoV-2, and then investigated the same abilities using live virus in vitro. Both CA1 and CB6 inhibit pseudovirus transduction into Huh7, Calu-3 and HEK293T cells (Fig. 2a-c). Notably, CB6 had stronger neutralizing activity than CA1 in terms of its $50 \%$ neutralization dose $\left(\mathrm{ND}_{50}\right)$ in all three tested cell lines. Consistently, $\mathrm{CB} 6$ also exhibited stronger neutralizing activity than CA1 against live SARS-CoV-2 infection of Vero E6 cells, with observed $N_{50}$ values of $0.036 \pm 0.007 \mu \mathrm{g} \mathrm{ml}^{-1}$ for CB6 and $0.38 \mu \mathrm{g} \mathrm{ml}^{-1}$ for CA1 (Fig. 2d, e). In summary, both mAbs exhibit substantial neutralizing activities against SARS-CoV-2 infection in vitro, although CB6 exhibited greater neutralizing activity than CA1.

We further tested CB6 in vivo in a rhesus macaque model of SARS-CoV-2 infection, in both prophylactic and treatment settings. Given the potential risk of an antibody-dependent enhancement effect (as observed in SARS-CoV infection) ${ }^{19,20}$, we introduced two leucine-to-alanine substitutions at residues 234 and 235 (known as 


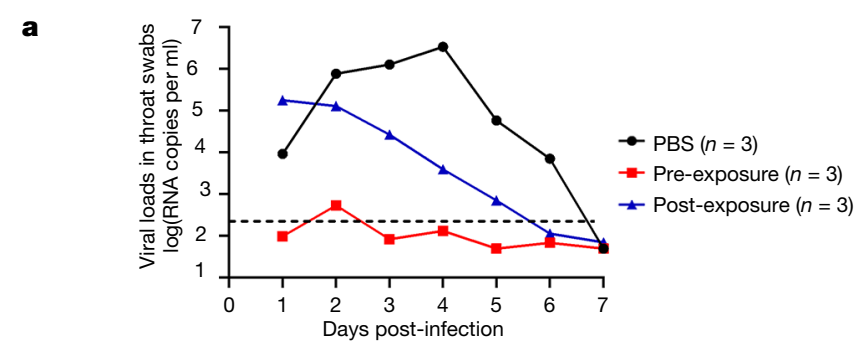

$\mathrm{H} \& \mathrm{E}$
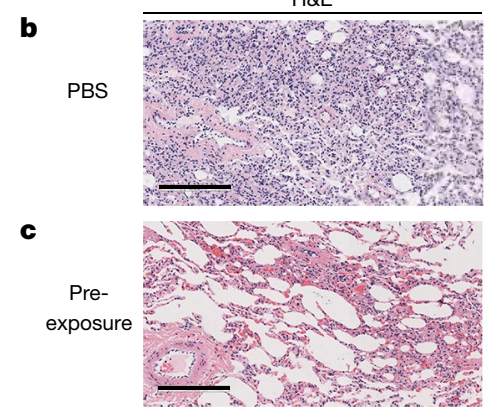

d

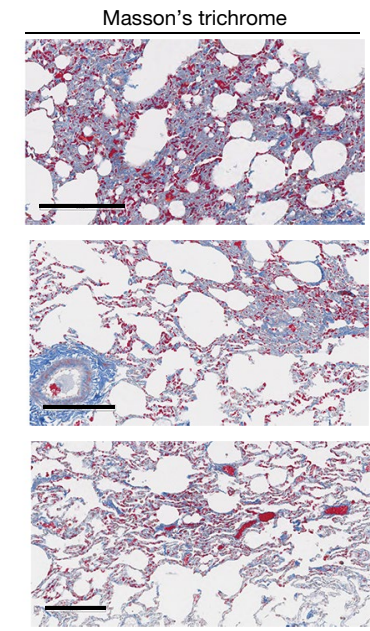

Fig. 3 | CB6 can effectively reduce viral load and alleviated infection-related lung damage in rhesus macaques. a, Nine rhesus macaques ( 6 males and 3 females) were divided into pre-exposure (prophylactic), post-exposure (treatment) and control groups with three macaques ( 2 males and 1 female) in each group. Before infection, the macaques in the pre-exposure group were infused with $50 \mathrm{mg} \mathrm{kg}^{-1}$ body weight CB6(LALA) intravenously. One day later, all macaques were inoculated with $1 \times 10^{5} \mathrm{TCID}_{50}$ SARS-CoV-2 via intratracheal intubation. The post-exposure group were also infused with $50 \mathrm{mg} \mathrm{kg}^{-1} \mathrm{CB} 6$ (LALA) on days 1 and 3 after exposure, and the three macaques in the control group were given PBS as a control. Viral RNA loads in throat swabs, determined by RT-qPCR, were monitored for 7 days. Data are average values from 3 macaques for the first 5 days, from 2 macaques at $6 \mathrm{dpi}$, and from 1 macaque at $7 \mathrm{dpi}$. To evaluate the viral loads for each macaque at the indicated time point, RT-qPCR were performed with two replicates. b-d, Histopathology and immunohistochemical examination of lung tissues from pre-exposure, post-exposure and control macaques. One macaque from each group was euthanized and necropsied at $5 \mathrm{dpi}$. Samples for histological examination were stored in formalin for 7 days, embedded in paraffin, sectioned and stained before examination by light microscopy. Haematoxylin and eosin (H\&E) sections exhibited the interstitial pneumonia and inflammatory-factor infiltration in tissues. Masson's trichrome showed lung tissue fibrosis. Scale bar, $200 \mu \mathrm{m}$.

the LALA mutation) of the Fc portion of CB6 (hereafter, CB6(LALA)) to lower the risk of $\mathrm{Fc}$-mediated acute lung injury. In the treatment group, three macaques (6-8 years old) were challenged with $1 \times 10^{5} 50 \%$ tissue culture infectious dose $\left(\mathrm{TCID}_{50}\right)$ of SARS-CoV-2 via intratracheal incubation. Fifty mg per kg body weight CB6(LALA) was administered at 1 and 3 days post-infection (dpi) intravenously. Macaques in the control group $(n=3)$ were given an equal volume of phosphate-buffered saline (PBS) at both 1 and $3 \mathrm{dpi}$. The viral titres from throat swabs were evaluated daily until $7 \mathrm{dpi}$. In the control group, the viral loads reached peak levels (approximately $10^{6.5} \mathrm{RNA}$ copies per $\mathrm{ml}$ ) at $4 \mathrm{dpi}$ and then declined naturally (Fig. 3a). By contrast, CB6(LALA) treatment reduced virus titres immediately after administration. Specifically, by 4 dpi, CB6(LALA) had reduced the viral titre by approximately $3 \log$ compared to the control group (Fig. 3a). Additionally, in the prophylactic group of three macaques, a single dose of CB6(LALA) $\left(50 \mathrm{mg} \mathrm{kg}^{-1}\right.$ body weight) before challenge with SARS-CoV-2 protected the macaques from SARS-CoV-2 infection. Only minimal levels of virus were detected in the throat swabs in this group and the peak viral load was no more than $10^{3}$ RNA copies per $\mathrm{ml}$ (Fig. 3a), indicating a strong prophylactic protection effect of CB6(LALA) antibody against infection with SARS-CoV-2.

In addition to reductions in viral titre, we further investigated whether CB6(LALA) also inhibited the pathological lung damage in the macaques challenged with SARS-CoV-2. One macaque from each group was euthanized and necropsied at $5 \mathrm{dpi}$. The control macaque displayed interstitial pneumonia characterized by thickened alveolar septa and proliferation and fibrosis of fibroblasts, with intensive infiltration of monocytes and lymphocytes. In some alveolar cavities, cellulose exudation was observed, with the formation of hyaline membrane and pulmonary haemorrhage. There was also clear thrombosis in the pulmonary capillary lumen, with accumulation of necrotic and exfoliated epithelial cells of the bronchioles (Fig. 3b). By contrast, macaques treated prophylactically or after infection displayed limited pathological lung damage. The necropsied macaques from the treatment and prophylactic groups had an overall intact alveolar structure, reduced oedema and no formation of hyaline membrane, with less fibrosis and less leukocyte infiltration compared to the control macaques (Fig.3c,d). In addition, no serious small bronchi and pulmonary capillary lesions were observed (Fig. 3c,d). Thus, CB6(LALA) inhibited SARS-CoV-2 viral titre and reduced infection-related lung damage, in both prophylactic and treatment settings.

To further investigate the blocking and neutralizing mechanisms of CB6, we prepared a protein complex of recombinant CB6 Fab and SARS-CoV-2 RBD for crystal screening (Extended Data Fig. 2). The 3D structure of the CB6 Fab-SARS-CoV-2 RBD complex was determined by X-ray crystallography at an overall resolution of $2.9 \AA$ (Extended Data Table 3). Overall, CB6 binds to SARS-CoV-2 RBD with a buried surface of $1,088 \AA^{2}$ (Fig. $4 a$ ). Specifically, the CB $6 V_{H}$ segment dominates the interaction with the SARS-CoV-2 RBD by all three CDRs, forming concentrated polar contacts (Fig. 4b, Extended Data Table 4) and hydrophobic interactions (Fig. 4c, Extended Data Table 4), whereas $V_{L}$ has limited contacts with the LCDR1 and LCDR3 loops (Fig. 4d, Extended Data Table 4).

To analyse the mechanisms by which CB6 blocks the interaction between SARS-CoV-2 RBD and ACE2, we superimposed the structure of the CB6 Fab-SARS-CoV-2 RBD complex with that of ACE2-SARS-CoV-2 RBD (Protein Data Bank (PDB) code 6LZG) (Fig. 4e). The superimposition of the RBD in its CB6-bound form with its ACE2-bound form resulted in $C \alpha$ root mean squared deviation of $0.282 \AA$ (for 169 atoms), indicating that the binding of CB6 did not induce substantial conformational changes in the SARS-CoV-2 RBD. The binding of CB6 induced steric hindrance to the binding of ACE2, which is mediated by both the $\mathrm{V}_{\mathrm{H}}$ and $\mathrm{V}_{\mathrm{L}}$ of CB6 (Fig. 4e). The whole of the CB6 light chain-as well as most of the heavy chain-has structure clashes with the receptor. There are also substantial overlapping binding areas between $\mathrm{CB} 6$ and ACE2 on the RBD (Fig. 4f). In summary, the blocking mechanisms of CB6 depends on both its $V_{H}$ and its $V_{L}$, which provide steric hindrance and direct competition for interface residues to abrogate the binding of ACE2 to the SARS-CoV-2 RBD.

Previous studies focusing on SARS-CoV infection in vitro and in mouse models ${ }^{19,20}$ indicate the potential risk of antibody-dependent enhancement hindering the ability of antibody-based therapies to control inflammation in the lung and other organs. On the contrary, antibody-dependent enhancement may lead to acute respiratory injury, acute respiratory distress syndrome and other observed inflammation-based sequelae. Given the close phylogenetic relationship between SARS-CoV and SARS-CoV-2, the risk of antibody-dependent enhancement needs to be mitigated. To solve this problem, we introduced the LALA mutations to the Fc portion of CB6 molecule to eliminate the antibody-dependent cellular cytotoxicity effect. Subsequently, CB6(LALA) showed a protective effect in a rhesus monkey model of SARS-CoV-2 infection, and did not exacerbate tissue damage induced by the infection. To our knowledge, this represents 

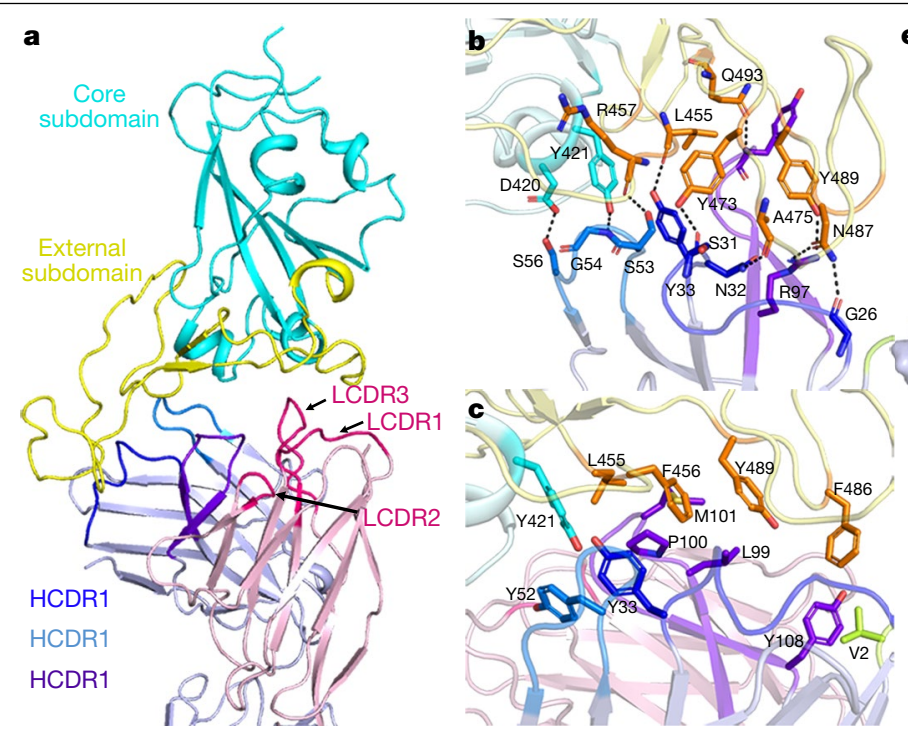

Fig. 4 | The crystal structure of the CB6-SARS-CoV-2 RBD complex and the competitive binding of CB6 and ACE2 with SARS-CoV-2 RBD. a, The structure of CB6 bound to SARS-CoV-2 RBD. The SARS-CoV-2 RBD is coloured cyan (core subdomain) and yellow (external subdomain). The variable fragment of $\mathrm{CB} 6$ is shown with HCDR1, HCDR2 and HCDR3 loops from the $V_{H}$ domain (purple) (which are coloured blue, marine and purple, respectively), and the LCDR1, LCDR2 and LCDR3 loops from the $V_{L}$ domain (pink) (which are coloured hot pink). b, c, Both hydrophilic interactions (b) and hydrophobic interactions (c) between the CB6 heavy chain and SARS-CoV-2 RBD are displayed. $d$, The binding details between the CB6 light chain and SARS-CoV-2 RBD are presented. The hydrogen

the first report to evaluate the function of neutralizing mAbs against SARS-CoV-2 using nonhuman primates, and can be used to inform the future design of dosing regimens in clinical trials.

Currently, no drugs or vaccines have been approved as a treatment for COVID-19, highlighting the unmet medical need to develop therapeutic agents. Neutralizing $m A b s$ are promising candidates to combat emerging viruses. Using Ebola virus as an example, ZMapp-which comprises three chimeric mAbs-was used to successfully treat two individuals with Ebola virus disease in $2014^{21}$; MAb114 has also shown notable treatment benefits, reducing the mortality rate of this disease from about $67 \%$ to $34 \%$ for all patients, and to $9.9 \%$ for patients with low viral loads ${ }^{22}$. Recently, MAb114 has been designated a breakthrough therapy for the treatment of Ebola virus disease. The neutralizing activity in vitro, and protection efficacy in vivo, of CB6 against SARS-CoV-2 is good and is likely to be comparable to that of MAb114 against Ebola virus $^{23,24}$.

In addition to post-exposure treatments, neutralizing mAbs can also be used in a prophylactic setting. The first mAb approved by the US Food and Drug Administration against infectious disease is palivizumab, which is indicated for the prophylaxis of pre-term infants and children at a high risk of infection with respiratory syncytia virus. The current pandemic of COVID-19 poses great risks to front-line healthcare workers, elderly patients and patients with pre-existing conditions. In the current situation (when vaccines are not available), preventative treatments are greatly needed. Our in vivo protection data from pre-exposure settings in macaques indicate that CB6 is a promising candidate for development as a prophylaxis for COVID-19.

Our structural analysis has revealed the molecular basis for the neutralizing activity of $\mathrm{CB} 6$. Notably, the interacting epitopes on the SARS-CoV-2 RBD for CB6 largely overlap with the binding epitopes of ACE2, which indicates that this $m A b$ has a strong resistance potential to SARS-CoV-2 mutation variants. In the 157 viral genomes for SARS-CoV-2 that are currently deposited in the National Center for Biotechnology Information (NCBI) databank (as of 1 April 2020), two substitutions in the RBD region have been observed (G476S and V483A) (Extended Data

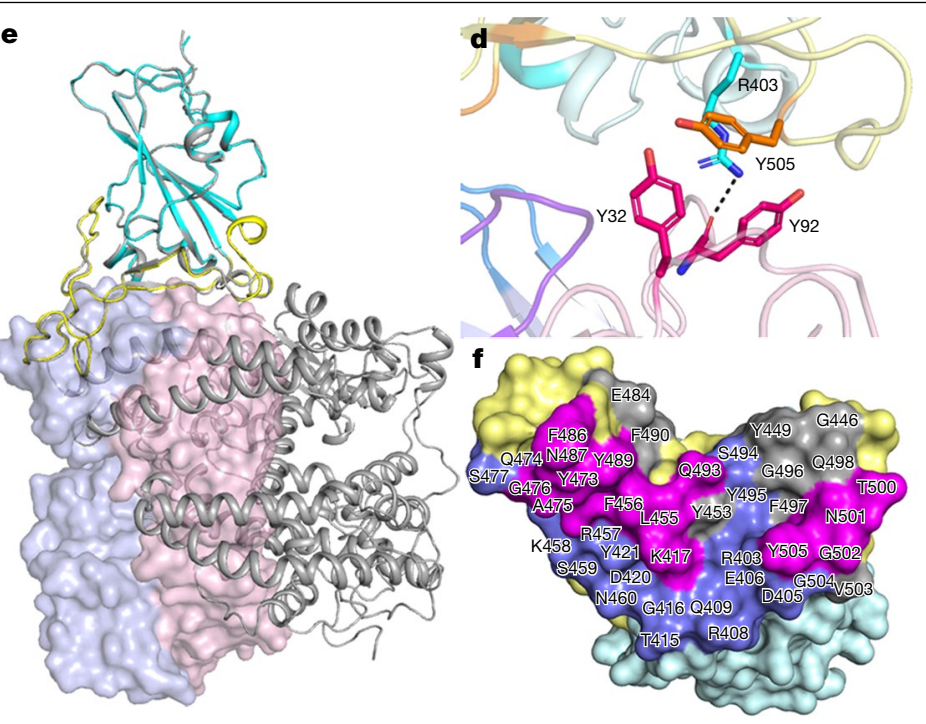

bonds are shown as dashed black lines. e, Superimposition of CB6-SARS-CoV-2 RBD complex and ACE2-SARS-CoV-2 RBD complex (PDB code 6LZG) reveals steric competition between CB6 and ACE2 for RBD binding. The CB6-SARS-CoV-2 RBD structure is superimposed on ACE2-SARS-CoV-2 RBD to demonstrate steric hindrance. ACE2 is shown as a cartoon (grey).f, Competitive binding surfaces of CB6 with ACE2 on SARS-CoV-2 RBD. The SARS-CoV-2 RBD binding surface to ACE2 and CB6 is shown. The residues bound by both CB6 and ACE2 are coloured magenta. The residues in contact with ACE2 alone are coloured grey, and the residues in contact with $\mathrm{CB} 6$ alone are coloured blue. The amino acids on the SARS-CoV-2 RBD interface that contact CB6 or ACE2 are labelled.

Fig. 3). Although G476S is located within the binding interface with CB6, this residue has limited contribution to the antibody-antigen interaction and is thus unlikely to influence the binding of CB6.

During the revision of this Article, CR3022-a neutralizing antibody against SARS-CoV that was previously isolated from a patient convalescing from SARS - was found to be cross-reactive with the SARS-CoV-2 RBD. Crystal-structure analysis revealed that CR3022 targets a highly conserved epitope, distal from the receptor-binding site and does not neutralize infection with SARS-CoV- ${ }^{25}$. Superimposition of the SARS-CoV-2 RBD in complex with either CB6 or CR3022 (PDB code $6 \mathrm{~W} 41$ ) indicates that it is unlikely that CB6 will have an effect on the binding of CR3022 (and vice versa) (Extended Data Fig. 4). Nevertheless, the reported molecular basis for the binding of CR3022 with SARS-CoV-2 RBD deepened our understanding of antibody recognition of SARS-CoV-2.

In conclusion, our data indicate that $\mathrm{CB} 6-$ a neutralizing $\mathrm{mAb}$ isolated from a patient convalescing from COVID-19-could be a potential therapeutic agent for the treatment of COVID-19 and that it deserves further translational development.

\section{Online content}

Any methods, additional references, Nature Research reporting summaries, source data, extended data, supplementary information, acknowledgements, peer review information; details of author contributions and competing interests; and statements of data and code availability are available at https://doi.org/10.1038/s41586-020-2381-y.

1. Tan, W. et al. Notes from the field: a novel coronavirus genome identified in a cluster of pneumonia cases - Wuhan, China 2019-2020. China CDC Weekly 2, 61-62 (2020).

2. The 2019-nCoV Outbreak Joint Field Epidemiology Investigation Team \& Li, Q. Notes from the field: an outbreak of NCIP (2O19-nCoV) infection in China - Wuhan, Hubei province, 2019-2020. China CDC Weekly 2, 79-80 (2020).

3. Wang, C., Horby, P. W., Hayden, F. G. \& Gao, G. F. A novel coronavirus outbreak of global health concern. Lancet 395, 470-473 (2020).

4. Coronaviridae Study Group of the International Committee on Taxonomy of Viruses. The species Severe acute respiratory syndrome-related coronavirus: classifying 2019-nCoV and naming it SARS-CoV-2. Nat. Microbiol. 5, 536-544 (2020). 


\section{Article}

5. Gao, G. F. From "A"IV to "Z"IKV: attacks from emerging and re-emerging pathogens. Cell 172, 1157-1159 (2018)

6. Zhou, P. et al. A pneumonia outbreak associated with a new coronavirus of probable bat origin. Nature 579, 270-273 (2020)

7. Jiang. S. et al. A distinct name is needed for the new coronavirus. Lancet 395, 949 (2020).

8. Hoffmann, M. et al. SARS-CoV-2 cell entry depends on ACE2 and TMPRSS2 and is blocked by a clinically proven protease inhibitor. Cell 181, 271-280.e8 (2020).

9. Lu, G., Wang, Q. \& Gao, G. F. Bat-to-human: spike features determining 'host jump' of coronaviruses SARS-CoV, MERS-CoV, and beyond. Trends Microbiol. 23, 468-478 (2015).

10. Wang, Q. et al. Structural and functional basis of SARS-CoV-2 entry by using human ACE2. Cell 181, 894-904.e9 (2020).

11. Shang, J. et al. Structural basis of receptor recognition by SARS-CoV-2. Nature 581, 221-224 (2020).

12. Lan, J. et al. Structure of the SARS-CoV-2 spike receptor-binding domain bound to the ACE2 receptor. Nature 581, 215-220 (2020).

13. Yan, R. et al. Structural basis for the recognition of SARS-CoV-2 by full-length human ACE2. Science 367, 1444-1448 (2020).

14. Li, Y. et al. A humanized neutralizing antibody against MERS-CoV targeting the receptor-binding domain of the spike protein. Cell Res. 25, 1237-1249 (2015).

15. Du, L. et al. The spike protein of SARS-CoV-a target for vaccine and therapeutic development. Nat. Rev. Microbiol. 7, 226-236 (2009).

16. Wang, Q., Wong, G., Lu, G., Yan, J. \& Gao, G. F. MERS-CoV spike protein: targets fo vaccines and therapeutics. Antiviral Res. 133, 165-177 (2016).

17. Wang, Q. et al. Molecular determinants of human neutralizing antibodies isolated from a patient infected with Zika virus. Sci. Transl. Med. 8, 369ra179 (2016).
18. Wang, Q. et al. Neutralization mechanism of human monoclonal antibodies against Rift Valley fever virus. Nat. Microbiol. 4, 1231-1241 (2019).

19. Channappanavar, R. et al. Dysregulated type I interferon and inflammatory monocyte-macrophage responses cause lethal pneumonia in SARS-CoV-infected mice. Cell Host Microbe 19, 181-193 (2016).

20. Liu, L. et al. Anti-spike IgG causes severe acute lung injury by skewing macrophage responses during acute SARS-CoV infection. JCl Insight 4, e123158 (2019).

21. Lyon, G. M. et al. Clinical care of two patients with Ebola virus disease in the United States. N. Engl. J. Med. 371, 2402-2409 (2014)

22. Mulangu, S. et al. A randomized, controlled trial of Ebola virus disease therapeutics. $N$. Engl. J. Med. 381, 2293-2303 (2019).

23. Gaudinski, M. R. et al. Safety, tolerability, pharmacokinetics, and immunogenicity of the therapeutic monoclonal antibody mAb114 targeting Ebola virus glycoprotein (VRC 608): an open-label phase 1 study. Lancet 393, 889-898 (2019).

24. Corti, D. et al. Protective monotherapy against lethal Ebola virus infection by a potently neutralizing antibody. Science 351, 1339-1342 (2016)

25. Yuan, M. et al. A highly conserved cryptic epitope in the receptor binding domains of SARS-CoV-2 and SARS-CoV. Science 368, 630-633 (2020).

26. Sui, J. et al. Potent neutralization of severe acute respiratory syndrome (SARS) coronavirus by a human $\mathrm{mAb}$ to $\mathrm{S} 1$ protein that blocks receptor association. Proc. Natl Acad. Sci. USA 101, 2536-2541 (2004).

Publisher's note Springer Nature remains neutral with regard to jurisdictional claims in published maps and institutional affiliations.

(c) The Author(s), under exclusive licence to Springer Nature Limited 2020 


\section{Methods}

\section{Study design}

This study was designed to isolate SARS-CoV-2-specific mAbs from the blood of patients recovering from infections with SARS-CoV-2; characterize the properties of mAbs, including blocking function, binding affinity, neutralization against SARS-CoV-2 and neutralizing mechanism; and then to test the best-performing $\mathrm{mAb}$ for therapeutic efficacy in rhesus macaques.

Peripheral blood mononuclear cells were isolated, following the manufacturer's instructions (GE Healthcare), before being subjected to cell sorting as previously reported ${ }^{17}$. In brief, peripheral blood mononuclear cells were incubated with His-tagged SARS-CoV-2 RBD at $100 \mathrm{nM}$ before staining with anti-CD3, anti-CD16, anti-CD235a, anti-CD19, anti-CD38, anti-CD27 and anti-His. Antigen-specific memory B cells were identified by the following markers: $\mathrm{CD}^{-}, \mathrm{CD}^{-} 6^{-}, \mathrm{CD} 235 \mathrm{a}^{-}, \mathrm{CD} 38^{-}, \mathrm{CD} 19^{+}$, $\mathrm{CD}^{2} 7^{+}, \mathrm{IgG}^{+}$and $\mathrm{His}^{+}$, and sorted into 96 -well $\mathrm{PCR}$ plates with single cell per well. Flow cytometric analysis and cell sorting were performed on a BD FACSAria III flow cytometer (BD Biosciences) and the data were analysed using Flowjo. The genes that encode the immunoglobulin $\mathrm{V}_{\mathrm{H}}$ and $\mathrm{V}_{\mathrm{L}}$ chains were amplified by $5^{\prime}$ RACE and nested PCR. The variable regions of these genes were then linked with the coding sequences for human IgG1 constant region to generate full-length mAbs, which were then expressed and purified under good laboratory practice conditions.

Eleven mAbs were generated with this method. Through blocking assays for SARS-CoV-2 RBD and ACE2 by FACS, two mAbs were analysed for several properties: (a) binding specificity for the $S$ protein of MERS-CoV, SARS-CoV and SARS-CoV-2; (b) the competition assay for mAbs binding using Octet; and (c) the ability of the mAbs to neutralize the SARS-CoV-2 in Vero E6 cells. The results suggested that these two mAbs specifically bound and potently neutralized SARS-CoV- 2 in vitro. Using a rhesus macaque model of SARS-CoV-2 infection, we tested the protective and curative efficacy of CB6. We equally divided the nine macaques into pre-exposure, post-exposure and negative-control groups. Viral loads from throat swabs were detected in the 3 groups over a consecutive period of 7 days. The macaques were euthanized and necropsied on $5 \mathrm{dpi}$ to evaluate the therapeutic effect of CB6. Then, we investigated the neutralizing mechanism of the mAb through structural analysis of CB6-SARS-CoV-2RBD. The high-resolution structure of this complex clarified that the binding surface of CB6 on the SARS-CoV-2 RBD overlapped with that of ACE2, indicating a substantial stereospecific hindrance to ACE2 contacting RBD.

\section{Cells and viruses}

HEK293T (ATCC, CRL-3216) cells, Huh7 (Institute of Basic Medical Sciences CAMS, 3111C0001CCC000679) cells, Calu-3 (ATCC, HTB-55) cells and Vero E6 (ATCC, CRL-1586) cells were cultured at $37^{\circ} \mathrm{C}$ in Dulbecco's Modified Eagle medium (DMEM) supplemented with $10 \%$ fetal bovine serum (FBS). The cells have not been identified or tested for mycoplasma contamination. The SARS-CoV-2 strain BetaCoV/Wuhan/IVDC-HB-envF13/2020 (accession identifier: EPI_ISL_408511) was isolated by the National Institute for Virus Disease Control and Prevention (Chinese Center for Disease Control and Prevention). Vero E6 cells were applied to the reproduction of SARS-CoV-2 stocks.

\section{Gene construction}

The recombinant proteins SARS-CoV-2 RBD and ACE2 were used in FACS, surface plasmon resonance (SPR), biolayer interferometry (BLI) and crystal screening assays. The coding sequences of SARS-CoV-2 RBD tagged with a C-terminal $6 \times$ His tag was cloned into the pCAGGS expression vector using the EcoRI and Xhol restriction sites. The coding sequences of ACE2 (residues 19-615, accession number BAJ21180) was cloned into the baculovirus transfer vector $\mathrm{pFastbac1}$ (Invitrogen) containing an $\mathrm{N}$-terminal gp67 signal peptide and a C-terminal $6 \times \mathrm{His}$ tag. The full-length coding region of the SARS-CoVS (accession number NC_004718), MERS-CoVS (accession number JX869050) or SARS-CoV-2 $S$ protein with a C-terminal Flag tag was cloned into the pCAGGS vector using the EcoRI and Smal restriction sites (pCAGGS-SARS-CoV-S-Flag, pCAGGS-MERS-CoV-S-Flag and pCAGGS-SARS-CoV-2-S-Flag, respectively). The pEGFP-N1-ACE2 plasmid was constructed by cloning the coding region of ACE2 into pEGFP-N1 using restriction enzymes Xhol and Smal.

\section{Protein expression and purification}

The SARS-CoV-2 RBD recombinant protein was expressed in HEK293T cells. The pCAGGS plasmid containing the SARS-CoV-2 RBD coding sequences was transiently transfected into cells. After 3 days, the supernatant was collected and soluble protein was purified by Ni affinity chromatography using a His Trap HP 5-ml column (GE Healthcare). The sample was further purified via gel filtration chromatography with a Superdex 200 column (GE Healthcare) in a buffer composed of $20 \mathrm{mM}$ Tris- $\mathrm{HCl}(\mathrm{pH} 8.0)$ and $150 \mathrm{mM} \mathrm{NaCl}$. The Bac-to-Bac baculovirus expression system (Invitrogen) was used to express the ACE2 for Octet analysis. The constructed pFastbac1 vectors were transformed into DH10Bac competent cells to generate recombinant bacmids. Transfection of bacmids and virus amplification were conducted in Sf 9 cells, and $\mathrm{Hi} 5$ cells were used for protein expression. The soluble ACE2 protein was purified by the same process as described for the purification of SARS-CoV-2 RBD.

\section{FACS assay}

The activity of mAbs to block the binding between the SARS-CoV-2 RBD and ACE 2 was assessed by FACS. HEK293T cells were transiently transfected with the pEGFP-N1-ACE2 expression plasmid for $24 \mathrm{~h}$. The SARS-CoV-2 RBD protein at a concentration of $200 \mathrm{ng} / \mathrm{ml}$ was mixed with the mAbs or isotype IgG at a molar ratio of 1:10 and incubated at $4{ }^{\circ} \mathrm{C}$ for $1 \mathrm{~h}$. Then mixtures were added to $5 \times 10^{5} \mathrm{HEK} 293 \mathrm{~T}$ cells expressing ACE2 and incubated at $4{ }^{\circ} \mathrm{C}$ for another hour. After washing with PBS three times, the cells were stained with anti-His APC-conjugated antibody for another $30 \mathrm{~min}$ and analysed using flow cytometry (BD FACSCalibur).

To test the binding specificity, pCAGGS-SARS-CoV-S-Flag, pCAGGS-MERS-CoV-S-Flag and pCAGGS-SARS-CoV-2-S-Flag plasmids were transfected into HEK293T cells using PEI (Alfa) according to the manufacturer's instructions. Four hundred thousand cells were collected $24 \mathrm{~h}$ after transfection, and suspended in permeabilization solution (BD) on ice for 20 min. After washing with PBS twice, the cells were incubated with $20 \mu \mathrm{g} / \mathrm{ml} \mathrm{CA1,} \mathrm{CB6,} \mathrm{80R,} \mathrm{4C2} \mathrm{or} \mathrm{isotype} \mathrm{IgG} \mathrm{at} \mathrm{room}$ temperature for $1 \mathrm{~h}$, followed by washing with PBS twice and further incubation with anti-human IgG APC-conjugated antibody (with the exception of 4C2, which used anti-mouse IgG APC-conjugated antibody as the second antibody) and anti-Flag FITC-conjugated antibodies. After washing, the cells were analysed using a BD FACSCalibur. All of these data were analysed using FlowJo.

\section{SPR}

SPR measurements were done at room temperature using a BIAcore $8 \mathrm{~K}$ system with Protein A biosensor chips (GE Healthcare). For all measurements, a buffer consisting of $150 \mathrm{mM} \mathrm{NaCl}, 10 \mathrm{mM}$ HEPES, pH 7.4 and $0.005 \%(\mathrm{v} / \mathrm{v}$ ) Tween-20 was used as running buffer, and all proteins were exchanged into this buffer in advance through gel filtration. The blank channel of the chip served as the negative control. Two mAbs were captured on the chip at 6,000 response units. Gradient concentrations of SARS-CoV-2 RBD (from $50 \mathrm{nM}$ to $3.125 \mathrm{nM}$ with twofold dilution) then flowed over the chip surface. After each cycle, the sensor was regenerated with $\mathrm{Gly}-\mathrm{HCl}(\mathrm{pH} 1.7)$. The affinity was calculated using a 1:1 (Langmuir) binding fit model with BIAevaluation software. 


\section{BLI}

The competitive binding of CA1, CB6 and ACE2 were measured by BLI using the Octet RED96 system (FortéBio). All experiments were performed at $25^{\circ} \mathrm{C}$ in a buffer containing $20 \mathrm{mM}$ Tris- $\mathrm{HCl}$ and $150 \mathrm{mM} \mathrm{NaCl}$, $\mathrm{pH}$ 7.0. Streptavidin biosensors were pre-equilibrated in the buffer for at least 10 min before use in experiments. Biotinylated SARS-CoV-2 RBD was loaded onto streptavidin biosensors for 300 s. For determining competitive characteristics, sensors were immersed with the first protein (or buffer as a control) for $240 \mathrm{~s}$, and then the second protein for another $240 \mathrm{~s}$. We analysed the data using FortéBio Data Analysis.

\section{Neutralization assay}

SARS-CoV-2 pseudovirus preparation was performed as previously described $^{27}$. In brief, the plasmids of $15 \mu$ g pCAGGS-SARS-CoV-2-S and $15 \mu \mathrm{g} \mathrm{pNL} 4-3$. luc.RE were cotransfected into HEK293T cells. Forty-eight $h$ later, the supernatant was sequentially collected, centrifuged and filtered through a $0.22 \mu \mathrm{m}$ sterilized membrane. The $\mathrm{TCID}_{50}$ was determined by the transduction of pseudovirus into Huh7 cells. For the neutralization assay, $10^{4}$ cells perwell in $100 \mu$ lwereseeded in the 96 -well plates $16 \mathrm{~h}$ before infection. Fifty $\mu$ l supernatant containing $10^{3} \mathrm{TCID}_{50}$ pseudovirus was incubated with equal volume of threefold serially diluted antibodies for $1 \mathrm{~h}$ at $37^{\circ} \mathrm{C}$. Both $\mathrm{mAbs}$ were tested in the concentrations ranging from $5.08 \mathrm{ng} / \mathrm{ml}$ to $100 \mu \mathrm{g} / \mathrm{ml}$ in the context of Huh7 cells and Calu-3 cells; for HEK293T cells, $15.24 \mathrm{ng} / \mathrm{ml}$ to $300 \mu \mathrm{g} / \mathrm{ml} \mathrm{mAb}$ concentrations were used. The mixtures of pseudoviruses and mAbs were then added to Huh7 cells, with two replicates. After a 4-h incubation, the medium was replaced with DMEM containing $10 \%$ fetal bovine serum, and the samples were incubated for an additional $24 \mathrm{~h}$ at $37^{\circ} \mathrm{C}$. Cell lysates were transferred $(10 \mu \mathrm{l}$ per well) into luminometer plates (Microfluor 96-well plates), and luciferase substrate (40 $\mu \mathrm{l}$ per well)-included in luciferase assay system-was added. Luciferase activity was measured using a GloMax 96 Microplate luminometer (Promega). The 50\% neutralization dose was calculated using GraphPad Prism 6.0.

An infectious SARS-CoV-2 neutralization assay was performed. Vero E6 cells were seeded at $8 \times 10^{4}$ per well in a 24 -well culture plates at $37^{\circ} \mathrm{C}$ for $24 \mathrm{~h}$ before being used. To reach a multiplicity of infection of $0.005,1 \mathrm{ml}$ of diluted SARS-CoV-2 virus and twofold serially diluted CA1 (from $5.0 \mu \mathrm{g} / \mathrm{ml}$ to $0.15 \mu \mathrm{g} / \mathrm{ml}$ ) or CB6 (from $1.0 \mu \mathrm{g} / \mathrm{ml}$ to $0.015 \mu \mathrm{g} / \mathrm{ml}$ ) mAbs were mixed in the medium containing $2 \% \mathrm{FBS}$. Meanwhile, a control group (without antibody) was set up, and the culture supernatant containing virus was collected $30 \mathrm{~h}$ post infection. One hundred and forty $\mu \mathrm{l}$ of the supernatant at each well was taken and RNA extraction was carried out according to the instructions of QIAamp viral RNA mini kit. RT-qPCR detection was carried out with one-step TB Green PrimeScript PLUS RT-PCR Kit (Perfect Real Time), and the primer was RBD-qF1: 5'-CAATGGTTTAACAGGCACAGG-3', RBD-qR1: 5'-CTCAAGTGTCTGTGGATCACG-3'. The total volume of the reaction system was $20 \mu \mathrm{l}$. The reaction conditions were: reverse transcription at $42^{\circ} \mathrm{C}$ for $5 \mathrm{~min}$, pre-denaturation at $95^{\circ} \mathrm{C}$ for $10 \mathrm{~s}, 40$ cycles including denaturation at $95^{\circ} \mathrm{C}$ for $10 \mathrm{~s}$, and annealing and extension at $60^{\circ} \mathrm{C}$ for $30 \mathrm{~s}$. Another neutralization assay was based on cytopathic effect (CPE). Serial twofold dilutions of $50 \mu \mathrm{l}$ of antibody CA1 (from $97.6 \mathrm{ng} / \mathrm{ml}$ to $200 \mu \mathrm{g} / \mathrm{ml}$ ) or CB6 (from $48.8 \mathrm{ng} / \mathrm{ml}$ to $100 \mu \mathrm{g} / \mathrm{ml}$ ) were prepared in a 96-well tissue culture plate in MEM medium. An equal volume of SARS-CoV-2 virus containing $100 \mathrm{TCID}_{50}$ was added, and the antibody-virus mixture was incubated at $37^{\circ} \mathrm{C}$ for $1 \mathrm{~h}$. The antibody-virus mixture was then transferred into a 96-well microtitre plate containing an equal volume of confluent Vero E6 cells with 8 repeats and incubated at $37^{\circ} \mathrm{C}$ for 3 days. Cells infected with 100 $\mathrm{TCID}_{50}$ of SARS-CoV-2 and without the virus were applied as positive and uninfected controls, respectively. The CPE in each well was observed daily and recorded on day 3 after infection. A virus back-titration was performed to assess the correct virus titre used in each experiment. The $\mathrm{ND}_{50}$ was calculated using GraphPad Prism 6.0. All experiments were followed the standard operating procedures of the approved Biosafety Level 3 facility.

\section{Human samples}

The human samples were obtained according to procedures approved by the Chinese Academy of Sciences, and complied with all relevant ethical regulations regarding human research. The blood was taken from a patient convalescing from COVID-19 after they had signed the informed consent form.

\section{Macaque experiments}

All macaque experiments were carried out according to the procedures approved by the Chinese Academy of Sciences and complied with all relevant ethical regulations regarding animal research.

Nine rhesus macaques (6-8 years old, 3 females and 6 males) were inoculated with $1 \times 10^{5} \mathrm{TCID}_{50}$ SARS-CoV-2 via intratracheal routes. The rhesus macaques were divided into three groups: a control group (one female and two males), a pre-exposure group (one female and two males) and a post-exposure group (one female and two males). The rhesus macaques in the control group were injected with PBS. The pre-exposure group were given $50 \mathrm{mg} / \mathrm{kg} \mathrm{CB} 6$ intravenously one day before viral challenge, and the post-exposure group were injected with the same amount of antibody on days 1 and 3 after exposure. In addition to routine clinical observation, oropharyngeal swabs were collected for seven days. Swabs were placed into $1 \mathrm{ml}$ of DMEM after collection. Viral RNA was extracted using the QIAamp Viral RNA Mini Kit (Qiagen), according to the manufacturer's instructions, and was eluted in $50 \mu \mathrm{l}$ of elution buffer and used as the template for RT-qPCR. The pairs of primers were used targeting the $S$ gene: RBD-qF1: 5'-CAATGGTTTAACAGGCACAGG-3'; RBD-qR1: 5'-CTCAAGTGTCTGTGGATCACG-3'. Two $\mu$ I RNA was used to verify the RNA quantity by HiScript II One Step qRT-PCR SYBR Green Kit (Vazyme Biotech) according to the manufacturer's instructions. The amplification was performed as followed: $50^{\circ} \mathrm{C}$ for $3 \mathrm{~min}, 95^{\circ} \mathrm{C}$ for $30 \mathrm{~s}$ followed by 40 cycles consisting of $95^{\circ} \mathrm{C}$ for $10 \mathrm{~s}, 60^{\circ} \mathrm{C}$ for $30 \mathrm{~s}$, and a default melting curve step in an $\mathrm{ABI}$ StepOne machine.

\section{Histopathology and immunohistochemistry}

Macaque necropsies were performed according to a standard protocol. Samples for histological examination were stored in $10 \%$ neutral-buffered formalin for 7 days, embedded in paraffin, sectioned and stained with haematoxylin and eosin or Masson's trichrome before examination by light microscopy.

\section{Crystal screening and structure determination}

The SARS-CoV-2 RBD protein and CB6 Fab fragment were mixed at a molar ration of 1:1. The mixture was incubated on ice for $1 \mathrm{~h}$ and further purified by Superdex-200 column (GE Healthcare). Five $\mathrm{mg} / \mathrm{ml}$ and $10 \mathrm{mg} / \mathrm{ml}$ of stable complex formed by SARS-CoV-2 RBD and CB6 Fab proteins, respectively, were used for crystal screening by vapour-diffusion sitting-drop method at $18^{\circ} \mathrm{C}$. Diffracting crystals were obtained in a concentration of $5 \mathrm{mg} / \mathrm{ml}$ at the mother liquid containing $24 \% \mathrm{w} / \mathrm{v}$ PEG $1500,20 \% \mathrm{v} / \mathrm{v}$ glycerol.

For data collection, the crystals were cryo-protected by briefly soaking in reservoir solution supplemented with $20 \%$ (v/v) glycerol before flash-cooling in liquid nitrogen. Diffraction data were collected at Shanghai Synchrotron Radiation Facility BL17U1 (wavelength, $0.97919 \AA$ ) at $100 \mathrm{~K}$. The dataset was processed with XDS software (from W. Kabsch, http://xds.mpimf-heidelberg.mpg.de/). The structure of the SARS-CoV-2 RBD-CB6 complex was determined by the molecular replacement method using Phaser $^{28}$ with the previously reported SARS-CoV-2 RBD structure (PDB code 6LZG) and Fab structure (PDB code 4TSA). The atomic models were completed with $\operatorname{Coot}^{29}$ and refined with phenix.refine in Phenix ${ }^{30}$, The Ramachandran plot determined by MolProbity indicates that $96.3 \%$ in the most-favoured region, $3.53 \%$ in the allowed region and $0.16 \%$ in the disallowed region ${ }^{31}$. Data collection, processing, and refinement statistics are summarized in Extended Data 
Table 3. All structural figures were generated using Pymol software (http://www.pymol.org).

\section{Sequence alignments}

The GenBank accession number of the sequences used for analysing the conservation of epitopes among SARS-CoV-2 are the following: MT263459, MT262993, MT262907, MT262915, MT262909, MT262916, MT262911, MT262908, MT262913, MT262912, MT262914, MT262906, MT262899, MT262898, MT262897, MT262904, MT262905, MT262896, MT262903, MT262902, MT262901, MT262900, MT262910, NC_045512, MT263396, MT263445, MT263421, MT258381, MT263391, MT263446, MT259269, MT263395, MT263468, MT263381, MT263074, MT258379, MT259271, MT263458, MT263429, MT258380, MT263382, MT259228, MT259227, MT259231, MT259226, MT259254, MT258377, MT259248, MT258383, MT263435, MT263398, MT258382, MT259277, MT263392, MT263418, MT259236, MT263406, MT263417, MT263436, MT263443, MT259256, MT263452, MT259264, MT263420, MT259281, MT263450, MT263402, MT263433, MT263413, МT263404, МT259260, MT263437, MT263438, MT263403, MT263399, MT263464, MT259261, MT263439, MT259237, MT263410, MT259251, MT263444, MT263465, MT263447, MT263454, MT263463, MT263431, MT263424, MT263432, MT263416, MT259282, MT263419, MT263467, MT263440, MT263411, MT256917, MT256918, MT256924, MT263405, MT263408, MT263386, MT263469, MT263400, MT263425, MT263387, MT259285, MT263430, MT263449, MT263412, MT263414, MT263462, MT263434, MT263457, MT263461, MT263448, MT263423, MT263422, MT263415, MT259249, MT263442, MT259245, MT263427, MT263384, MT263441, MT263383, MT259243, MT263426, MT263388, MT263428, MT263455, MT263456, MT263453, MT263451, MT259235, MT263460, MT263390, MT263394, MT263466, MT259250, MT259280, MT259258, MT259239, MT259240, MT263407, MT263385, MT263397, MT263409, MT263393, MT263401, MT259279, MT259284, MT263389, MT259270, MT259259, MT259242 and MT259238. The sequence logos were generated using the web server of Weblogo (http://weblogo.berkeley.edu/logo.cgi).

\section{Reporting summary}

Further information on research design is available in the Nature Research Reporting Summary linked to this paper.

\section{Data availability}

Further information and requests for resources and reagents should be directed toJ.Y. The accession number for the atomic coordinates and diffraction data reported in this study is $\mathrm{PDB}$ code 7C01. The sequences of CA1 and CB6 mAbs have been deposited in GenBank with the accession codes MT470194-MT470197. Source data are provided with this paper.

27. Du, L., Zhang, X., Liu, J. \& Jiang, S. Protocol for recombinant RBD-based SARS vaccines: protein preparation, animal vaccination and neutralization detection. J. Vis. Exp. 51, 2444 (2011).

28. McCoy, A. J. et al. Phaser crystallographic software. J. Appl. Crystallogr. 40, 658-674 (2007).

29. Emsley, P., Lohkamp, B., Scott, W. G. \& Cowtan, K. Features and development of Coot. Acta Crystallogr. D 66, 486-501 (2010).

30. Adams, P. D. et al. PHENIX: a comprehensive Python-based system for macromolecular structure solution. Acta Crystallogr. D 66, 213-221 (2010).

31. Chen, V. B. et al. MolProbity: all-atom structure validation for macromolecular crystallography. Acta Crystallogr. D 66, 12-21 (2010).

Acknowledgements We thank S. Yao for his help in revising the manuscript; the staff of beamline BL17U1 at the Shanghai Synchrotron Radiation Facility for assistance during data collection; Z. Fan for her technical help in performing BLI and SPR; all colleagues of the National Biosafety Laboratory for their support during this study; Y. Yao and Y. Peng for supporting all the experiments in nonhuman primates; and F. Wang, Y. Hu, Y. Jia, S. Ren, L. Wu and S. Tan for their help in performing the experiments. This work was supported by the Strategic Priority Research Program of CAS (XDB29040201), the National Major Science and Technology Major Project (2018ZX09711003), the National Key Research and Development Program of China (2020YFC0841400) and the National Natural Science Foundation of China (81922044 and 81973228). Q.W. is supported by the Youth Innovation Promotion Association CAS (2018119). G.F.G and J.Y are supported by the foundation of the NSFC Innovative Research Group (81621091).

Author contributions Q.W., G.F.G. and J.Y. initiated and coordinated the project. R.S., F.-S.W. Q.W., G.F.G., Z.Y. and J.Y. designed the experiments. Q.W. performed the cell sorting with the help of Z.C., J.S. and Z.T. R.S., X.D., T.S. and C.H. sequenced the antibodies. R.S. and X.D. conducted the SPR and Octet analysis. W.H. and L.W. prepared the pseudotype SARS-CoV-2 virus. T.S. evaluated the neutralizing potency using pseudovirus. Y.Z. and P.L. evaluated the neutralizing potency using authentic virus, with the help from B.Z., W.J.L. and G.W. C.S. conducted the animal experiments. Y.Z. and Z.Y. performed histopathology and immunohistochemistry assays with the assistance from G.G. and X.H. X.D., X.B. and C.H. grew the complex crystals. J.Q. collected the diffraction data and determined the complex structure with the help from L.W. R.S., H.F., F.-S.W., Q.W., G.F.G. and J.Y. analysed the data and wrote the manuscript.

Competing interests R.S., Q.W., G.F.G. and J.Y. are listed as inventors on pending patent applications for $\mathrm{CA} 1$ and $\mathrm{CB} 6$. The pending patent of $\mathrm{CB} 6$ has been licensed. The other authors declare no competing interests.

Additional information

Supplementary information is available for this paper at https://doi.org/10.1038/s41586-0202381-y.

Correspondence and requests for materials should be addressed to F.-S.W., Q.W., G.F.G., Z.Y. or J.Y.

Reprints and permissions information is available at http://www.nature.com/reprints. 


\section{Article}

a

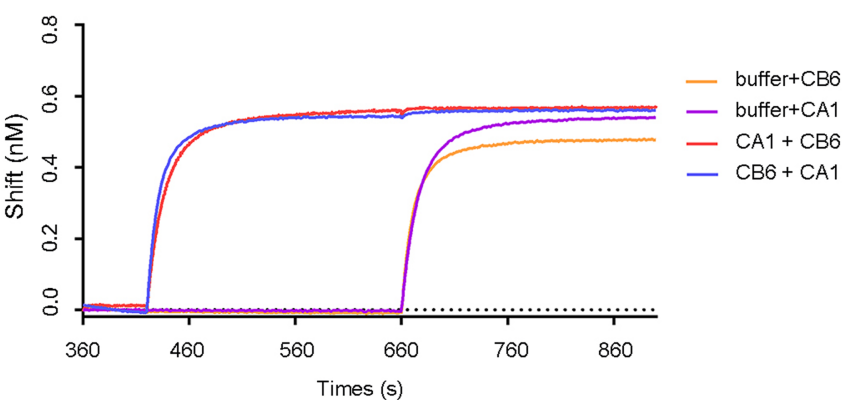

Extended Data Fig. 1 | Analysis of direct competitive binding characteristics of CA1, CB6 and ACE2. a, b, Competition between CA1 and CB6 (a) or either mAb and ACE2 (b) for binding to the SARS-CoV-2 RBD. Octet sensors immobilized with the SARS-CoV-2 RBD were first saturated with one mAb or the kinetic buffer, and b

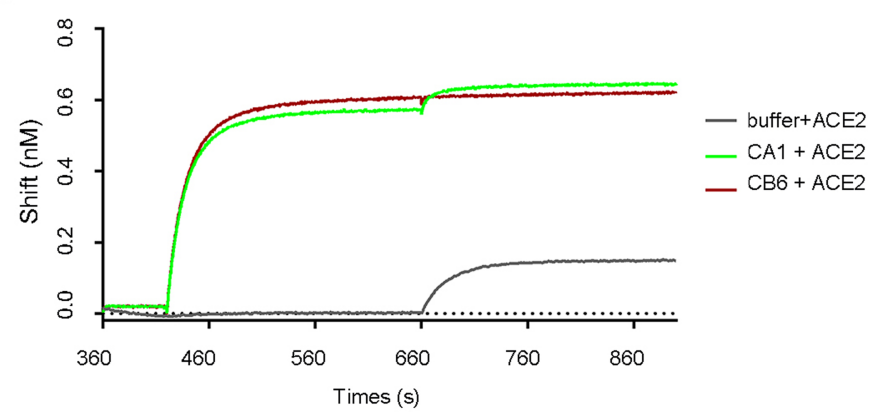

then exposed to the other mAb or ACE2 protein. The experiments were independently performed twice and similar results were obtained. The binding profiles of one experiment are shown. 

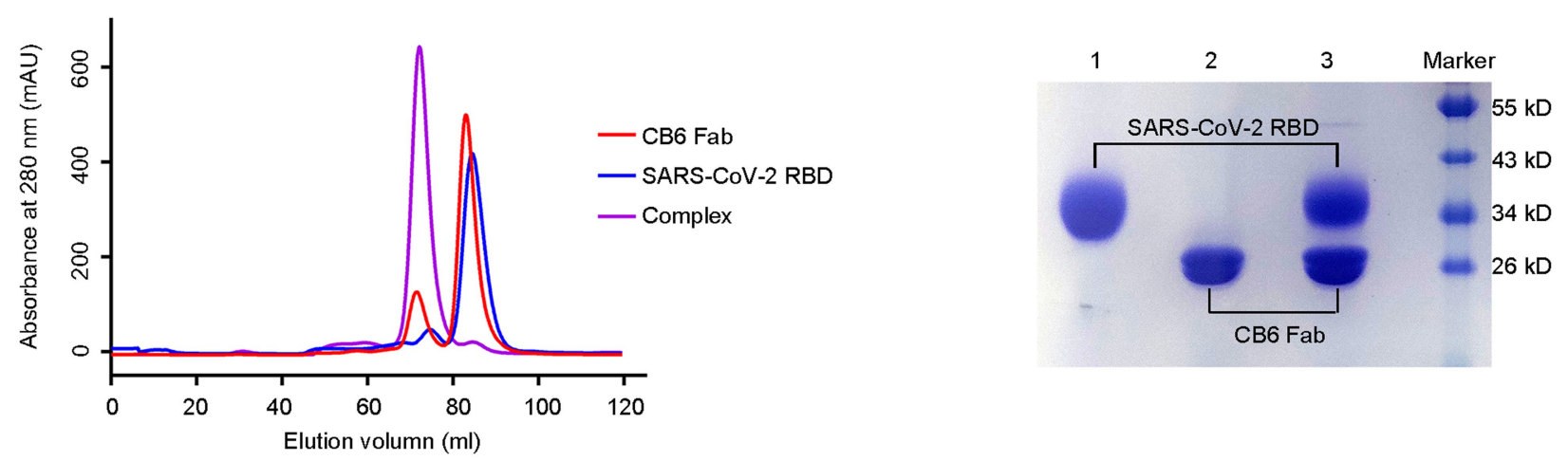

Extended Data Fig. 2 | Preparation of the CB6 Fab-SARS-CoV-2 RBD protein complex. a, Gel filtration profiles of CB6 Fab (red), SARS-CoV-2 RBD (blue) and the complex of the two (purple) were analysed by size-exclusion chromatography as indicated. The CB6 Fab forms a stable complex with the SARS-CoV-2 RBD. The experiments were independently performed twice and similar results were

obtained. b, The SDS-PAGE analyses are shown in reducing (+DTT) conditions. The CB6 Fab was depolymerized into $V_{H}$ and $V_{L}$ fragments $(25 \mathrm{kDa})$. The SARS-CoV-2 RBD exhibited a diffuse band, owing due to glycosylation ( $38 \mathrm{kDa})$. For gel source data, see Supplementary Fig. 1 . 


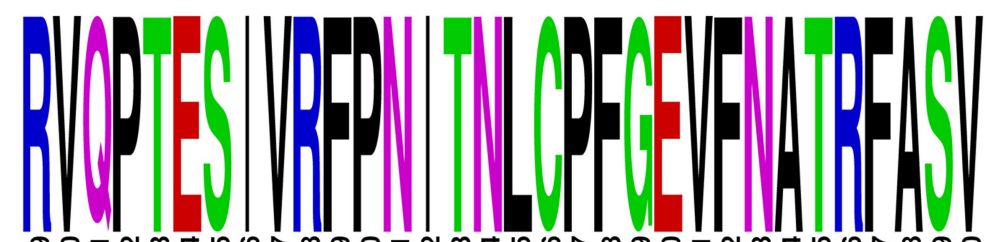

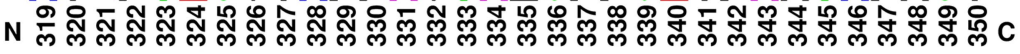

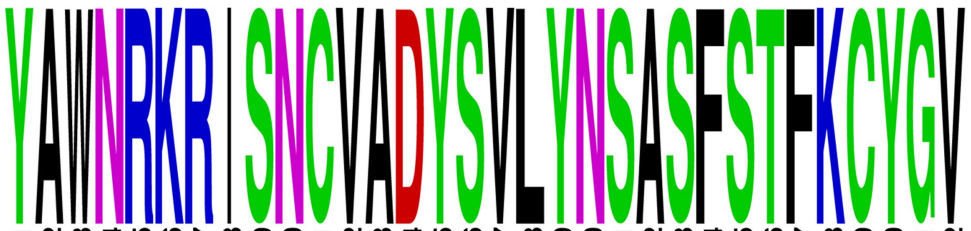

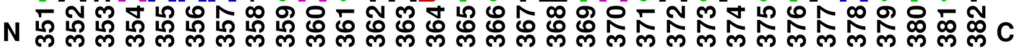

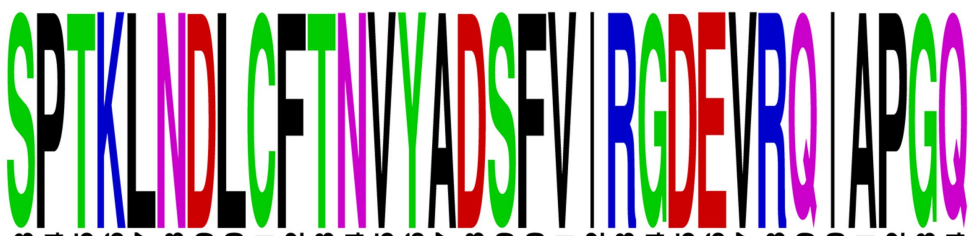

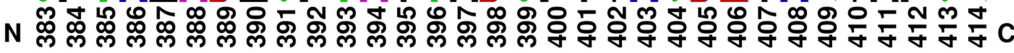
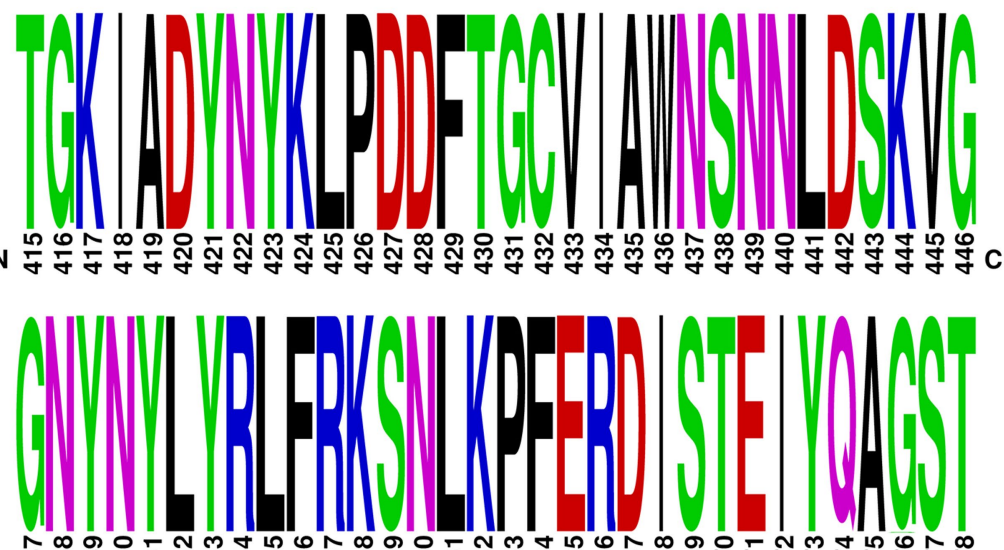

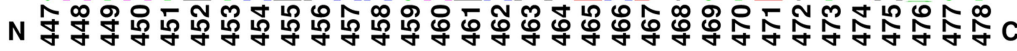
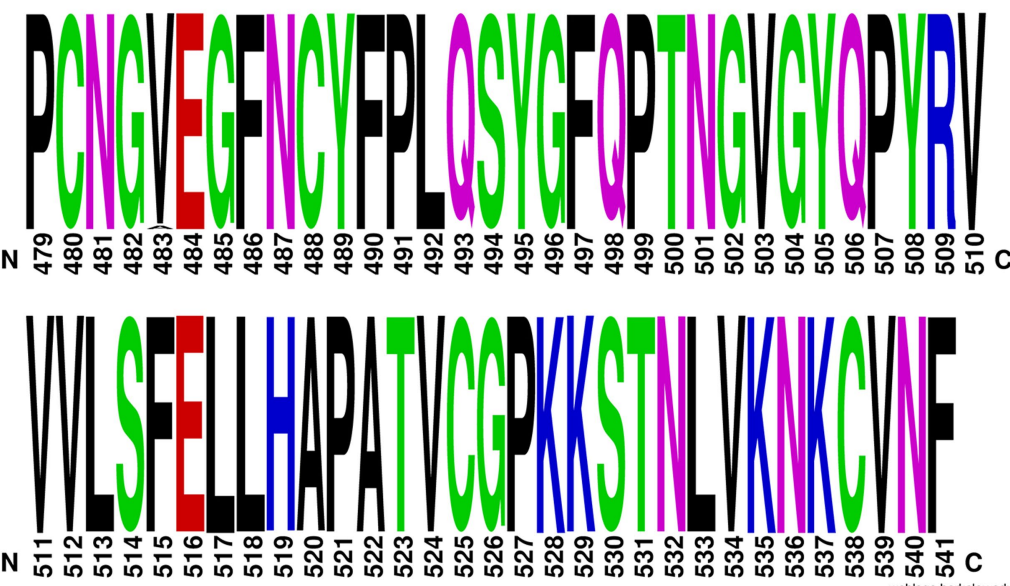

Extended Data Fig. 3 | Sequence alignment of RBD among strains of SARS-CoV-2. We obtained 157 full-length sequences of the SARS-CoV-2 RBD by searching the NCBI databank. The sequence logos were generated using the
Weblogo web server (http://weblogo.berkeley.edu/logo.cgi). Four strains isolated from human patients with COVID-19 in the USA carry G476S, and eight strains carry V483A; further details are provided in Methods. 


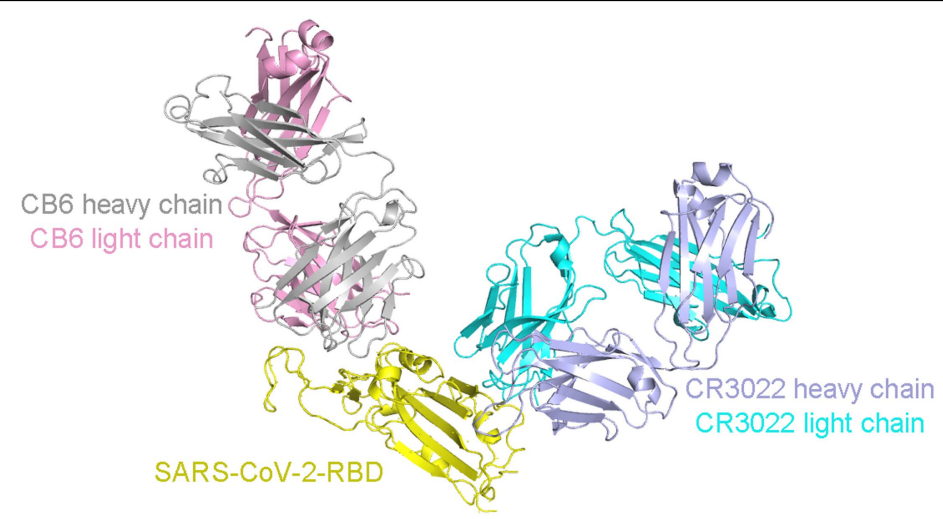

Extended Data Fig. 4 | Comparison of the binding epitopes of CB6 and CR3022. Superimposition of the CB6-SARS-CoV-2 RBD and CR3022SARS-CoV-2 RBD (PDB code 6W41) complexes reveals no epitope overlapping between CB6 and CR 3022. The SARS-CoV-2 RBD is shown as a cartoon (yellow).
The CB6 heavy chain and light chain are coloured grey and pink, respectively. The CR3022 heavy chain and light chain are coloured light blue and cyan, respectively. 
Article

Extended Data Table 1 | Germline analysis of CA1 and CB 6

\begin{tabular}{cccccccccc}
\hline & V-H allele & D-H allele & J-H allele & Identity $(V-H)$ & CDR3-H (aa) & V-L allele & J-L allele & Identity (V-L) & CDR3-L (aa) \\
\hline CA & IGHV1-18*01 & IGHD2-15*01 & IGHJ6*02 & $99.7 \%$ & AREGYCSGGSCYSGYYYYYGMDV & IGKV3-11*01 & IGKJ3*01 & $98.2 \%$ & QQRRNWGT \\
CB6 & IGHV3-66*01 & IGHD3-10*02 & IGHJ4*02 & $99.0 \%$ & ARVLPMYGDYLDY & IGKV1-39*01 & IGKJ2*01 & $99.6 \%$ & QQSYSTPPEYT \\
\hline
\end{tabular}

$V(D) J$ summary for the isolated CA 1 and CB6. The top matches for Homo sapiens germline genes corresponding to the V, D and J segments of the heavy chain ( $V$ - $H$, J-H and D-H) and V and J regmints of the light chain (V-L and J-L) are listed. The CDR3 heavy chain (CDR3-H) and light chain (CDR3-L) show the amino acid (aa) sequences of CDR3 for both chains. 
Extended Data Table 2 | Binding characteristics of CA1 and CB6

\begin{tabular}{llll}
\hline & $\mathrm{Ka}(1 / \mathrm{Ms})$ & $\mathrm{Kd}(1 / \mathrm{s})$ & $K_{\mathrm{D}}(\mathrm{M})$ \\
\hline CA1 & $3.98 \mathrm{E}+06$ & $1.16 \mathrm{E}-02$ & $2.92 \mathrm{E}-09$ \\
& $4.58 \mathrm{E}+05$ & $3.15 \mathrm{E}-03$ & $6.87 \mathrm{E}-09$ \\
& $8.66 \mathrm{E}+05$ & $3.69 \mathrm{E}-03$ & $4.26 \mathrm{E}-09$ \\
$\mathrm{~N}$ CB6 & $8.95 \mathrm{E}+05$ & $7.29 \mathrm{E}-04$ & $8.15 \mathrm{E}-10$ \\
& $2.32 \mathrm{E}+05$ & $4.49 \mathrm{E}-04$ & $1.93 \mathrm{E}-09$ \\
& $4.72 \mathrm{E}+05$ & $2.23 \mathrm{E}-04$ & $4.73 \mathrm{E}-09$ \\
\hline
\end{tabular}

The binding characteristics of CA1 and CB6 to the SARS-CoV-2 RBD. The results from three independent experiments are listed. $K_{\mathrm{a}}$ is the binding rate of the indicated $\mathrm{mAb}$ with the SARS-CoV-2 RBD; $K_{d}$ is the dissociation rate; and $K_{D}$ is the affinity of the indicated $m A b$ and the antigen. 


\section{Article}

Extended Data Table 3 | Data collection and refinement statistics

\begin{tabular}{ll}
\hline & CB6/SARS-CoV-2-RBD \\
\hline $\begin{array}{l}\text { Data collection } \\
\text { Space group }\end{array}$ & P212121 \\
Cell dimensions & \\
$\quad a, b, c(\AA)$ & $87.40,106.73,170.59$ \\
$\quad \alpha, \beta, \gamma\left({ }^{\circ}\right)$ & $90.00,90.00,90.00$ \\
Resolution $(\AA)$ & $50.00-2.88(2.95-2.88)^{*}$ \\
$R_{\text {sym } \text { or } R_{\text {merge }}}$ & $0.200(1.596)$ \\
$I / \sigma I$ & $12.2(1.8)$ \\
Completeness $(\%)$ & $99.9(100.0)$ \\
Redundancy & $13.0(13.6)$ \\
& \\
Refinement & \\
Resolution $(\AA)$ & $34.83-2.88$ \\
No. reflections & 36767 \\
$R_{\text {work }} / R_{\text {free }}$ & $0.2183 / 0.2663$ \\
No. atoms & \\
$\quad$ Protein & 9670 \\
Ligand/ion & 0 \\
$\quad$ Water & 0 \\
$B$-factors & \\
Protein & \\
Ligand/ion & \\
Water & \\
R.m.s. deviations & \\
Bond lengths $(\AA)$ & \\
Bond angles $\left({ }^{\circ}\right)$ & \\
\hline & \\
\hline
\end{tabular}

Diffraction data from one crystal were used for structure determination. *Values in parentheses are for the highest-resolution shell. 
Extended Data Table 4 | Residues that contribute to the interaction between CB6 and the SARS-CoV-2 RBD or ACE2 and the SARS-CoV-2 RBD

\begin{tabular}{|c|c|c|}
\hline SARS-CoV-2-RBD & CB6 & hACE2 \\
\hline R403 & Y92 ${ }^{*}\left(12,2^{\dagger}\right)$, T94 (3) & \\
\hline D405 & Y92 (1), T94 (7) & \\
\hline E406 & T94 (4) & \\
\hline R408 & T94 (4), P95 (13) & \\
\hline Q409 & T94 (2) & \\
\hline T415 & S56 (5), F58 (5) & \\
\hline G416 & Y52 (2), F58 (2) & \\
\hline K417 & Y33 (4), Y52 (7), D140 (4) & $\mathrm{D} 30(4,1)$ \\
\hline D420 & Y52 (1), S56 (6) & \\
\hline Y421 & Y33 (6), Y52 (8), S53 (8, 2), G54 (7, 1), G55 (1) & \\
\hline G446 & & Q42 (4) \\
\hline Y449 & & D38 (9), Q42 $(4,1)$ \\
\hline Y453 & & $\mathrm{H} 34(5,1)$ \\
\hline L455 & Y33 (4, 1), P100 (10), M101 (5) & D30 (2), K31 (2), H34 (9) \\
\hline F456 & S31 (2), Y33 (3), S53 (1), P100 (8), M101 (2) & T27 (5), D30 (4), K31 (5) \\
\hline R457 & S53 (8), G54 $(2,1)$ & \\
\hline K458 & S31 (3), S53 (3), G54 (2) & \\
\hline S459 & G54 (1) & \\
\hline N460 & G54 (11), G55 (1), S56 $(3,1)$ & \\
\hline Y473 & $\mathrm{S} 31(13,1), \mathrm{S} 53(2)$ & T27 (1) \\
\hline Q474 & S31 (2) & \\
\hline A475 & F27 (3), T28 (8, 1), S31 (3), N32 (8, 1), R97 (1) & S19 (3), Q24 (4), T27 (2) \\
\hline G476 & F27 (2), T28 (5) & S19 (4), Q24 (5) \\
\hline S477 & T28 (2) & \\
\hline E484 & & $\mathrm{K} 31(1)$ \\
\hline F486 & V2 (3), G26 (2), R97 (3), Y108 (7) & L79 (2), M82 (9), Y83 (11) \\
\hline N487 & $\mathrm{G} 26(4,1), \mathrm{F} 27(5), \mathrm{N} 32(1), \mathrm{R} 97(4,1)$ & Q24 $(15,1), Y 83(8,1)$ \\
\hline Y489 & R97 (3, 1), L99 (8), M101 (5) & T27 (7), F28 (7), K31 (6), Y 83 (1) \\
\hline F490 & & K31 (2) \\
\hline Q493 & M101 (4), Y102 $(6,1)$ & K31 (3), H34 (6), E35 (11, 1) \\
\hline Y495 & Y32 (1) & \\
\hline G496 & & D38 (5), K353 (7, 1), \\
\hline Q498 & & D38 (1), Y41 (8), Q42 (8, 1), L45 (3) \\
\hline \multirow[t]{2}{*}{ T500 } & & Y41 (7, 1), L45 (1), N330 (8), D355 (8), \\
\hline & & R357 (3) \\
\hline N501 & S30(1) & Y41 $(8,1), \mathrm{K} 353(11)$ \\
\hline G502 & S28 (1), Y92 (4) & K353 (4, 1), G354 (7), D355 (1) \\
\hline G504 & Y92 (3) & \\
\hline \multirow[t]{2}{*}{ Y505 } & S30 (4), Y32 $(13,1), Y 92(9,1)$ & E37 (7), K353 (28), G354 (4), R393 (1) \\
\hline & Total $321 \ddagger$ & Total 291 \\
\hline
\end{tabular}

*Italics represent the residues from CB6 light-chain CDRs.

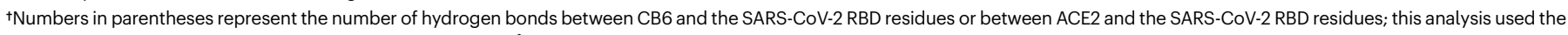
Contact program in the CCP4 suite (the distance cut-off is $3.5 \AA$ ).

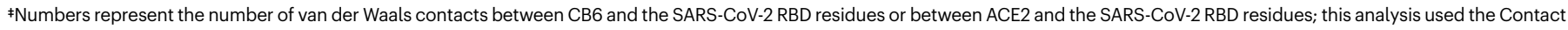
program in the CCP4 suite (the distance cut-off is $4.5 \AA$ ). 


\section{natureresearch}

Corresponding author(s): George Fu Gao

Last updated by author(s): May 16, 2020

\section{Reporting Summary}

Nature Research wishes to improve the reproducibility of the work that we publish. This form provides structure for consistency and transparency in reporting. For further information on Nature Research policies, see Authors \& Referees and the Editorial Policy Checklist.

\section{Statistics}

For all statistical analyses, confirm that the following items are present in the figure legend, table legend, main text, or Methods section.

n/a Confirmed

$\mathbf{X}$ The exact sample size $(n)$ for each experimental group/condition, given as a discrete number and unit of measurement

X A statement on whether measurements were taken from distinct samples or whether the same sample was measured repeatedly

$\mathbf{X} \square$ The statistical test(s) used AND whether they are one- or two-sided

$X$ Only common tests should be described solely by name; describe more complex techniques in the Methods section.

$\mathbf{X} \square$ A description of all covariates tested

$\mathbf{X} \square$ A description of any assumptions or corrections, such as tests of normality and adjustment for multiple comparisons

$x$ A full description of the statistical parameters including central tendency (e.g. means) or other basic estimates (e.g. regression coefficient)

AND variation (e.g. standard deviation) or associated estimates of uncertainty (e.g. confidence intervals)

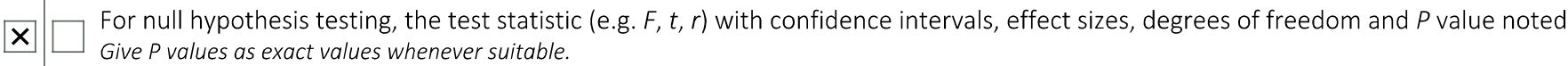

$\mathbf{X} \square$ For Bayesian analysis, information on the choice of priors and Markov chain Monte Carlo settings

$\mathbf{X} \square$ For hierarchical and complex designs, identification of the appropriate level for tests and full reporting of outcomes

$\mathbf{X} \square$ Estimates of effect sizes (e.g. Cohen's $d$, Pearson's $r$ ), indicating how they were calculated

Our web collection on statistics for biologists contains articles on many of the points above.

\section{Software and code}

Policy information about availability of computer code

Data collection

BD FACSDiva Software 8.0.3, Biacore 8K Control Software 2.0.15.12933, GLOMAX 1.9.3, Octet Data Acquisition 9.0.0.26, UNICIORN 7.0.0.953 and XDS Program Package (Jan 31,2020).

Data analysis

FlowJo 7.6.1, Biacore Insight Evaluation 1.0.5.11069, GraphPad Prism 6.01, CCP4 7.0.072, Coot 0.8.9, Phenix 1.10.1-2155, Pymol 2.3.3 and ForteBio Data Analysis 9.0.0.10

For manuscripts utilizing custom algorithms or software that are central to the research but not yet described in published literature, software must be made available to editors/reviewers. We strongly encourage code deposition in a community repository (e.g. GitHub). See the Nature Research guidelines for submitting code \& software for further information.

\section{Data}

Policy information about availability of data

All manuscripts must include a data availability statement. This statement should provide the following information, where applicable:

- Accession codes, unique identifiers, or web links for publicly available datasets

- A list of figures that have associated raw data

- A description of any restrictions on data availability

The accession number for the atomic coordinates and diffraction data reported in this study is PDB code 7C01. The sequences of CA1 and CB6 MAbs have been deposited in GenBank with the accession codes MT470194- MT470197. 


\section{Life sciences study design}

All studies must disclose on these points even when the disclosure is negative.

Sample size No statistical methods were used to predetermine sample size. For the animal study, there are three groups investigated, including the control group without administration of MAbs $(n=3)$, prophylactic group $(n=3)$ and treatment group $(n=3)$. The numbers of monkeys in each group meet the requirement for statistical analysis (at least 3 for each group), which is sufficient given the excellent technical reproducibility.

Data exclusions No data were excluded.

Replication The NHP experiments were not repeated in BSL-3 lab.

Randomization We divided nine monkeys (three females and six males) into three groups, inluding the control group, the prophylactic group and the treatment group. Monkeys of the same sex were randomly divided into three groups.

Blinding The investigators were not blinded to allocation during experiments and outcome assessment. Data collection and analysis were performed by different people, the sample classification were replaced by simple marks during data analysis.

\section{Behavioural \& social sciences study design}

All studies must disclose on these points even when the disclosure is negative.

Study description Briefly describe the study type including whether data are quantitative, qualitative, or mixed-methods (e.g. qualitative cross-sectional, quantitative experimental, mixed-methods case study).

Research sample State the research sample (e.g. Harvard university undergraduates, villagers in rural India) and provide relevant demographic information (e.g. age, sex) and indicate whether the sample is representative. Provide a rationale for the study sample chosen. For studies involving existing datasets, please describe the dataset and source.

Sampling strategy Describe the sampling procedure (e.g. random, snowball, stratified, convenience). Describe the statistical methods that were used to predetermine sample size $O R$ if no sample-size calculation was performed, describe how sample sizes were chosen and provide a rationale for why these sample sizes are sufficient. For qualitative data, please indicate whether data saturation was considered, and what criteria were used to decide that no further sampling was needed.

Data collection

Provide details about the data collection procedure, including the instruments or devices used to record the data (e.g. pen and paper, computer, eye tracker, video or audio equipment) whether anyone was present besides the participant(s) and the researcher, and whether the researcher was blind to experimental condition and/or the study hypothesis during data collection.

Timing

Indicate the start and stop dates of data collection. If there is a gap between collection periods, state the dates for each sample cohort.

Data exclusions

If no data were excluded from the analyses, state so OR if data were excluded, provide the exact number of exclusions and the rationale behind them, indicating whether exclusion criteria were pre-established.

Non-participation State how many participants dropped out/declined participation and the reason(s) given OR provide response rate OR state that no participants dropped out/declined participation.

Randomization

If participants were not allocated into experimental groups, state so OR describe how participants were allocated to groups, and if allocation was not random, describe how covariates were controlled.

All studies must disclose on these points even when the disclosure is negative.

Study description

Briefly describe the study. For quantitative data include treatment factors and interactions, design structure (e.g. factorial, nested, 


\section{Ecological, evolutionary \& environmental sciences study design}

Research sample

Describe the research sample (e.g. a group of tagged Passer domesticus, all Stenocereus thurberi within Organ Pipe Cactus National Monument), and provide a rationale for the sample choice. When relevant, describe the organism taxa, source, sex, age range and any manipulations. State what population the sample is meant to represent when applicable. For studies involving existing datasets, describe the data and its source.

Sampling strategy

Note the sampling procedure. Describe the statistical methods that were used to predetermine sample size OR if no sample-size calculation was performed, describe how sample sizes were chosen and provide a rationale for why these sample sizes are sufficient.

Data collection

Describe the data collection procedure, including who recorded the data and how.

Timing and spatial scale

Indicate the start and stop dates of data collection, noting the frequency and periodicity of sampling and providing a rationale for these choices. If there is a gap between collection periods, state the dates for each sample cohort. Specify the spatial scale from which the data are taken

Data exclusions

If no data were excluded from the analyses, state so OR if data were excluded, describe the exclusions and the rationale behind them, indicating whether exclusion criteria were pre-established.

Reproducibility

Describe the measures taken to verify the reproducibility of experimental findings. For each experiment, note whether any attempts to repeat the experiment failed $O R$ state that all attempts to repeat the experiment were successful.

Randomization

Describe how samples/organisms/participants were allocated into groups. If allocation was not random, describe how covariates were controlled. If this is not relevant to your study, explain why.

Blinding

Describe the extent of blinding used during data acquisition and analysis. If blinding was not possible, describe why OR explain why blinding was not relevant to your study.

Did the study involve field work? $\square$ Yes $\square$ No

Field work, collection and transport

Field conditions

Describe the study conditions for field work, providing relevant parameters (e.g. temperature, rainfall).

Location

State the location of the sampling or experiment, providing relevant parameters (e.g. latitude and longitude, elevation, water depth).

Access and import/export

Describe the efforts you have made to access habitats and to collect and import/export your samples in a responsible manner and in compliance with local, national and international laws, noting any permits that were obtained (give the name of the issuing authority, the date of issue, and any identifying information).

Disturbance

\section{Reporting for specific materials, systems and methods}

We require information from authors about some types of materials, experimental systems and methods used in many studies. Here, indicate whether each material, system or method listed is relevant to your study. If you are not sure if a list item applies to your research, read the appropriate section before selecting a response.

Materials \& experimental systems

\begin{tabular}{l|l}
\hline n/a & Involved in the study \\
\hline & $\mathbf{X}$ Antibodies \\
\hline & $\mathbf{X}$ Eukaryotic cell lines \\
\hline $\mathbf{X}$ & $\mathbf{X}$ Palaeontology \\
\hline & $\mathbf{X}$ Animals and other organisms \\
\hline $\mathbf{X}$ & Human research participants \\
\hline
\end{tabular}

Methods n/a Involved in the study

$\mathbf{x} \square$ ChIP-seq

$\square$ Flow cytometry

$\mathbf{X} \square$ MRI-based neuroimaging

\section{Antibodies}

Antibodies used

anti-His/PE: Miltenyi Biotec, Cat No: 130-120-718, Clone No. GG11-8F3.5.1, Lot No. 5200110309, Dilution: 1:10; anti-hCD3/PE-Cy ${ }^{\mathrm{TM}} 5$ : BD Pharmingen ${ }^{\mathrm{TM}}$, Cat No: 555334, Clone No. UCHT1, Lot No. 7037531, Dilution: 1:20; anti-hCD16/PE-Cy $^{\text {TM} 5: ~ B D ~ P h a r m i n g e n ~}{ }^{\text {TM }}$, Cat No: 555408, Clone No. 3G8, Lot No. 7045714, Dilution: 1:20; 
anti-hCD19/APC-Cy 7 : BD Pharmingen ${ }^{\mathrm{TM}}$, Cat No: 557791, Clone No. SJ25C1, Lot No. 7118676, Dilution: 1:20; anti-hCD27/Pacific Blue ${ }^{\mathrm{TM}}$ : BioLegend, Cat No: 302821, Clone No. O323, Lot No. B223941, Dilution: 1:50; anti-hCD38/APC: BD Pharmingen ${ }^{\mathrm{TM}}$, Cat No: 555462, Clone No. HIT2, Lot No. 7125887, Dilution: 1:20; anti-CD235a/PE-CY' 5 : BD Pharmingen ${ }^{\mathrm{TM}}$, Cat No: 559944, Clone No. GA-R2, Lot No. 7012580, Dilution: 1:20; anti-hlgG/FITC: BD Pharmingen ${ }^{\mathrm{TM}}$, Cat No: 555786, Clone No. G18-145, Lot No. 6363638, Dilution: 1:20; anti-hlgG/APC: BioLegend, Cat No: 409306, Clone No. HP6017, Lot No. B265810, Dilution: 1:100. Anti-mlgG/APC: BD Pharmingen ${ }^{\mathrm{TM}}$, Cat No: 550874, Clone No. X56, Lot No. 7128657, Dilution: 1:100.

Validation

We follow the manufactures's instruction to use the above listed antibodies to stain human PBMCs. All antibodies work well. anti-His/PE: (Miltenyi Biotec; Mouse; specific for His tag; applicable for Flow cytometry/Cell sorting [FC/FACS]) https://www.citeab.com/antibodies/7058497-130-120-718-anti-his-pe?des=d6e817455032c7cc

anti-hCD3/PE-Cy ${ }^{\mathrm{TM}}$ 5: (BD Pharmingen ${ }^{\mathrm{TM}}$; Mouse BALB/c IgG1, k; specific for Human [QC Testing] CD3; applicable for flow cytometry [Routinely Tested]; $20 \mu \mathrm{L} /$ test)

https://www.bdbiosciences.com/us/applications/research/t-cell-immunology/th-1-cells/surface-markers/human/pe-cy5-mouseanti-human-cd3-ucht1-also-known-as-ucht-1-ucht-1/p/555334

anti-hCD16/PE-Cy ${ }^{\mathrm{TM}} 5$ : (BD Pharmingen ${ }^{\mathrm{TM}}$; Mouse BALB/C x DBA/2, also known as CD2F1 or CDF1 IgG1, $\mathrm{k}$; specific for Human [QC Testing], Rhesus, Cynomolgus, Baboon [Tested in Development] CD16; applicable for Flow cytometry [Routinely Tested]; $20 \mu \mathrm{L} /$ test).

https://www.bdbiosciences.com/us/applications/research/stem-cell-research/cancer-research/human/pe-cy5-mouse-antihuman-cd16-3g8/p/555408

anti-hCD19/APC-Cy ${ }^{\text {TM}}$ : (BD Pharmingen ${ }^{\text {TM }}$, Mouse BALB/C IgG1, k; specific for Human [QC Testing] CD19; applicable for flow cytometry [Routinely Tested], $5 \mu \mathrm{L} /$ test)

https://www.bdbiosciences.com/us/applications/research/clinical-research/oncology-research/blood-cell-disorders/surfacemarkers/human/apc-cy7-mouse-anti-human-cd19-sj25c1-also-known-as-sj25-c1/p/557791

anti-hCD27/Pacific Blue ${ }^{\mathrm{TM}}$ : (BioLegend, Mouse IgG1, k; specific for Human, African Green, Baboon, Cynomolgus, Rhesus, Squirrel Monkey CD27; applicable for flow cytometry [Quality tested], $5 \mu \mathrm{L} /$ test)

https://www.biolegend.com/en-us/products/pacific-blue-anti-human-cd27-antibody-6442

anti-hCD38/APC: (BD Pharmingen ${ }^{\mathrm{TM}}$, Mouse BALB/c IgG1, k; specific for Human [QC Testing] CD19; applicable for flow cytometry [Routinely Tested], $20 \mu \mathrm{L} /$ test)

https://www.bdbiosciences.com/us/applications/research/t-cell-immunology/regulatory-t-cells/surface-markers/human/apcmouse-anti-human-cd38-hit2/p/555462

anti-CD235a/PE-Cy ${ }^{\text {TM}}$ 5: (BD Pharmingen ${ }^{\mathrm{TM}}$, Mouse IgG2b, k; specific for Human [QC Testing] CD19; applicable for flow cytometry [Routinely Tested])

https://www.bdbiosciences.com/us/reagents/research/antibodies-buffers/immunology-reagents/anti-human-antibodies/cellsurface-antigens/pe-cy5-mouse-anti-human-cd235a-ga-r2-hir2/p/559944

\section{Eukaryotic cell lines}

Policy information about cell lines

Cell line source(s)

HEK293T cells: ATCC, CRL-3216;

Huh7 cells: Institute of Basic Medical Sciences CAMS, 3111C0001CCC000679;

Calu-3 cells: ATCC, HTB-55;

Vero E6 cells: ATCC, CRL-1586.

Authentication

The cell lines were not authenticated since they were purchased commercially and are not commonly misidentified.

Mycoplasma contamination

The cells were not tested for mycoplasma contamination.

Commonly misidentified lines

(See ICLAC register)

The cell lines used in this study do not appear on the ICLAC register.

\section{Palaeontology}

Specimen provenance

Provide provenance information for specimens and describe permits that were obtained for the work (including the name of the issuing authority, the date of issue, and any identifying information).

Specimen deposition

Indicate where the specimens have been deposited to permit free access by other researchers.

Dating methods

If new dates are provided, describe how they were obtained (e.g. collection, storage, sample pretreatment and measurement) where they were obtained (i.e. lab name), the calibration program and the protocol for quality assurance OR state that no new dates are provided.

Tick this box to confirm that the raw and calibrated dates are available in the paper or in Supplementary Information. 
Animals and other organisms

Policy information about studies involving animals; ARRIVE guidelines recommended for reporting animal research

Laboratory animals

Nine Rhesus macaques (6-8 years old).

Wild animals

The study did not involve wild animals.

Field-collected samples

The study did not involve samples collected from the field.

Ethics oversight

Chinese Academy of Sciences

Note that full information on the approval of the study protocol must also be provided in the manuscript.

\section{Human research participants}

Policy information about studies involving human research participants

Population characteristics We used the blood from one convalescent COVID-19 patient (female, 33 years old) in China.

Recruitment

The patient agreed to provide the biospecimen for detection, further diagnostic and scientific research when hospitalization.

Ethics oversight

Chinese Academy of Sciences

Note that full information on the approval of the study protocol must also be provided in the manuscript.

\section{Clinical data}

Policy information about clinical studies

All manuscripts should comply with the ICMJE guidelines for publication of clinical research and a completed CONSORT checklist must be included with all submissions.

Clinical trial registration

Provide the trial registration number from ClinicalTrials. gov or an equivalent agency.

Study protocol

Note where the full trial protocol can be accessed OR if not available, explain why.

Data collection

Describe the settings and locales of data collection, noting the time periods of recruitment and data collection.

Outcomes

Describe how you pre-defined primary and secondary outcome measures and how you assessed these measures.

\section{ChIP-seq}

Data deposition

$\square$ Confirm that both raw and final processed data have been deposited in a public database such as GEO.

$\square$ Confirm that you have deposited or provided access to graph files (e.g. BED files) for the called peaks.

Data access links

May remain private before publication.

Files in database submission

Genome browser session (e.g. $\underline{\cup C S C})$

\section{Methodology}

Replicates

Sequencing depth

Antibodies

Peak calling parameters

Data quality
For "Initial submission" or "Revised version" documents, provide reviewer access links. For your "Final submission" document, provide a link to the deposited data.

Provide a list of all files available in the database submission.

Provide a link to an anonymized genome browser session for "Initial submission" and "Revised version" documents only, to enable peer review. Write "no longer applicable" for "Final submission" documents.
Describe the experimental replicates, specifying number, type and replicate agreement.

Describe the sequencing depth for each experiment, providing the total number of reads, uniquely mapped reads, length of reads and whether they were paired-or single-end.

Describe the antibodies used for the ChIP-seq experiments; as applicable, provide supplier name, catalog number, clone name and lot number.

Specify the command line program and parameters used for read mapping and peak calling, including the ChIP, control and index files used.

Describe the methods used to ensure data quality in full detail, including how many peaks are at FDR 5\% and above 5-fold enrichment. 


\section{Flow Cytometry}

Plots

Confirm that:

$\mathbf{X}$ The axis labels state the marker and fluorochrome used (e.g. CD4-FITC).

X The axis scales are clearly visible. Include numbers along axes only for bottom left plot of group (a 'group' is an analysis of identical markers).

X All plots are contour plots with outliers or pseudocolor plots.

X A numerical value for number of cells or percentage (with statistics) is provided.

Methodology

Sample preparation

Sample preparation listed in Methods

Instrument

BD FACSAria II, BD FACSCalibur and BD FACSCanto II.

Software

FlowJo 7.6.1

Cell population abundance After cell sorting, the collected cells were subjected for RT-PCR to determine the genes of the IgG. Thus, we did not test the relevant cell populations within post-sort fractions.

Gating strategy

For the isolation of antigen-specific memory B cells, we used the unstained PBMCs as control to gate cells.

$\mathbf{X}$ Tick this box to confirm that a figure exemplifying the gating strategy is provided in the Supplementary Information.

\section{Magnetic resonance imaging}

\section{Experimental design}

Design type

Design specifications

Behavioral performance measures

Acquisition

Imaging type(s)

Field strength

Sequence \& imaging parameters

Area of acquisition

Diffusion MRI

Used

Preprocessing

Preprocessing software

Normalization

Normalization template

Noise and artifact removal
Indicate task or resting state; event-related or block design.

Specify the number of blocks, trials or experimental units per session and/or subject, and specify the length of each trial o block (if trials are blocked) and interval between trials.

State number and/or type of variables recorded (e.g. correct button press, response time) and what statistics were used to establish that the subjects were performing the task as expected (e.g. mean, range, and/or standard deviation across subjects). 
Volume censoring

Statistical modeling \& inference

Model type and settings

Specify type (mass univariate, multivariate, RSA, predictive, etc.) and describe essential details of the model at the first and second levels (e.g. fixed, random or mixed effects; drift or auto-correlation).

Effect(s) tested

Define precise effect in terms of the task or stimulus conditions instead of psychological concepts and indicate whether ANOVA or factorial designs were used.

Specify type of analysis:

Whole brain

ROI-based

Both

Statistic type for inference

Specify voxel-wise or cluster-wise and report all relevant parameters for cluster-wise methods.

(See Eklund et al. 2016)

Correction

Describe the type of correction and how it is obtained for multiple comparisons (e.g. FWE, FDR, permutation or Monte Carlo).

Models \& analysis

n/a $\mid$ Involved in the study

$\square \square$ Functional and/or effective connectivity

$\square$ Graph analysis

$\square \square$ Multivariate modeling or predictive analysis

Functional and/or effective connectivity

Report the measures of dependence used and the model details (e.g. Pearson correlation, partial correlation, mutual information).

Graph analysis

Report the dependent variable and connectivity measure, specifying weighted graph or binarized graph, subject-or group-level, and the global and/or node summaries used (e.g. clustering coefficient, efficiency, etc.).

Multivariate modeling and predictive analysis

Specify independent variables, features extraction and dimension reduction, model, training and evaluation metrics. 